\title{
Preparation of Azulenes Substituted at Seven-Membered Cycle with 2- and 3-thiophenevinyl Groups
}

\author{
LIVIU BIRZAN*, VICTORITA TECUCEANU, CONSTANTIN C. DRAGHICI, \\ ANAMARIA HANGANU, ALEXANDRU C. RAZUS \\ Romanian Academy, Institute of Organic Chemistry, 202B Splaiul Independentei, 060023, Bucharest, Romania
}

\begin{abstract}
This paper comes as a continuation of our efforts to prepare vinylazulenes, moving our attention to the seven-membered azulene cycle. Several azulenic substrates were tested in order to study the possibility of preparation of poly(thiophenevinyl)azulenes, needed for the generation of dendrimeric structures. The compounds containing methyls in positions 4,6 and 8 could be condensed with 2-and 3thiophenecarbaldehyde in $\mathrm{tBuOK} / \mathrm{THF}$ yielding mono-, bis- and tris-condensed products in function of molar ratio of reagents, catalyst, solvent. The reaction works in thermodynamic control, preferring 6position instead of 4(8)-position, which generates less stable alkenes (steric hindrance) and has a trans stereochemistry. Usually mixtures of products are obtained if more condensation position are present, even if a large aldehyde excess is used. In forcing conditions, the intermediate alcohols suffer an Oppenauer hydride transfer to the excess aldehyde, generating undesired ketones. For condensation of a methyl group situated on the five-membered cycle it must be activated by substitution with ${ }^{+} P P h_{3}$ followed by a Wittig condensation.
\end{abstract}

Keywords: thiophenevinylazulenes, condensations, Wittig, H-NMR, UV-Vis spectra

\section{Introduction}

For a long time our research has focused on the synthesis and properties of azulene-containing compounds with (hetero)aryl- azo [1-5], imino [6] and ethene groups [7]. Most of the papers were reviewed as 1-vinylazulenes with aromatic and heteroaromatic moieties attached to the $\mathrm{C}=\mathrm{C}$ bond [8]. Other reviews treated them as separate chapters $[9,10]$. Some of the obtained compounds showed significant NLO properties [11], while others have interesting electrochemical properties [12]. In the literature, several attempts have also been made to bind arylvinyls to azulene seven-ring, such as, for example, the modified Heck reaction [13] between 6-bromoazulenes and styrene in the presence of $\mathrm{Pd}\left(\mathrm{PPh}_{3}\right)_{4}$. The condensation of the methyl group present on at seven-membered azulene ring with aldehydes was initiated by the German school. Scholtz and al. reported that 4-methylazulenes react with benzaldehydes in the presence of sodium N-methylanilide to produce 4-styrylazulenes in low yields (10$30 \%)$, the results being better $(80 \%)$ only for electron-rich benzaldehydes, which possess $\mathrm{p}-\mathrm{NMe}_{2}$ or $\mathrm{p}$ $\mathrm{OH}$ groups. In the case of guaiazulene for example, the main reaction products were the ketones resulting from the intermediate alcohol by hydride transfer to excess aldehydes ( 60\%) [14]. However, this transfer is prevented by an excess of anilide due to its reducing character, which changes the reaction course to generate mainly the benzylic alcohol. Subsequently, the Hansen group observed that by changing of the catalyst, the solvent and the reaction conditions, the condensation reaction of guaiazulene becomes more selective for the generation of the styryl compounds. In the presence of $\mathrm{KOtBu}$-TFA $\left(-5\right.$ to $\left.25^{\circ} \mathrm{C}\right)$, the yields of condensations with benzaldehydes increase up to $90 \%$. However, the reactions yields are very sensitive to steric requirements, falling to less than $20 \%$ if tert-butyl substituents are present on azulene moiety [15]. A more sophisticated study has been conducted in our days by Belfield's team, which presents quite different results of the condensation between guaiazulene and many aromatic aldehydes. Several catalysts with different basicities were tested, but the best results for benzaldehyde were obtained only for those containing t-BuOK. However, they were much smaller than those reported by Hansen [15]. No alkenic product was obtained for $\mathrm{K}_{2} \mathrm{CO}_{3}-\mathrm{DMF}, \mathrm{Cs}_{2} \mathrm{CO}_{3}-\mathrm{DMF}$,

\footnotetext{
*email:lbirzan@yahoo.com
} 
LDA-THF, while the desired alkene is generated in the presence of $\mathrm{tBuOK}$, the yields depending on the solvent: THF-29\%, THF-morpholine-40\%, t-AmOH-54\%, neat-48\% [16]. Instead of aldehydes, aromatic imines can be used, but even in this case a strong base, like $\mathrm{KOH}$, is needed as a catalyst. Briquet and Hansen have shown that the final step of aniline elimination is slow and therefore, the yields of alkenes depend heavily on the substituents of benzaldehyde Schiff bases. Yields decrease from $82 \%$ for $\mathrm{p}-\mathrm{NH}_{2}$ group to $0 \%$ for $\mathrm{p}-\mathrm{NO}_{2}$ [17]. In this context, we studied the behavior of 2-thiophenecarbaldehyde in these condensation conditions [18].
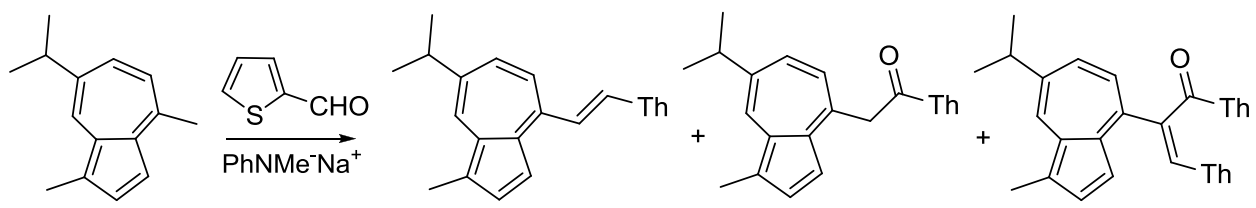

$19 \%$

$28 \%$

$26 \%$
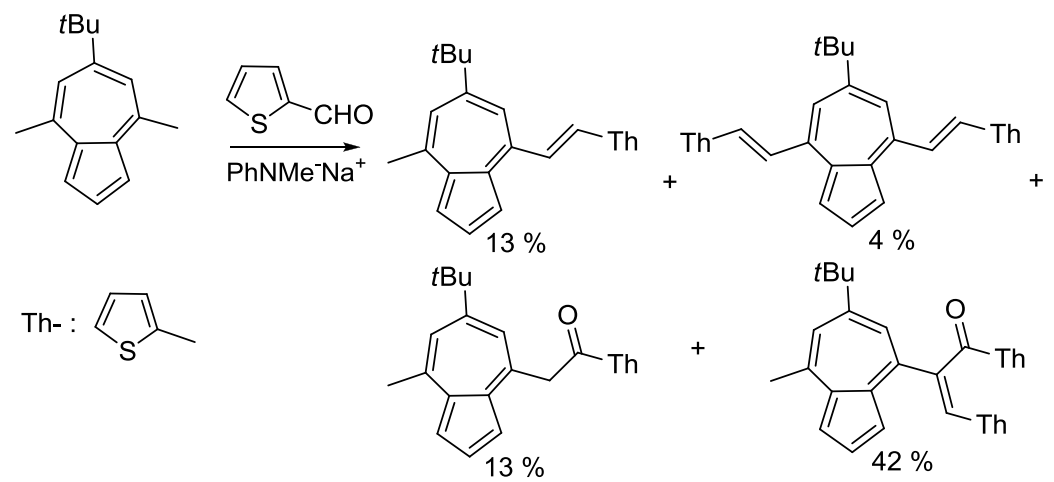

Scheme 1. Reactions between activated alkylazulenes and 2-carbaldehydes

In these reactions (Scheme 1), the carbanion resulting from the extraction of a proton from the methyl group, promoted by the base, reacts with aldehydes and, after water elimination, gives alkenes. The position of the reactive methyl belonging to guaiazulene, 4,6,8-trimethylazulene or 4,8-dimthyl-6-tertbutyl-azulene and the yields of resulted products depend on electronic, steric factors and reaction conditions. Thus, condensation of 4-Me group of guaiazulene with aryl carbaldehydes occurred with excellent yields in the presence of t-BuOK. However, when 4,8-dimethyl-6-tert-butyl-azulene was used, the mono vinyl derivative resulted only in $20 \%$ yield together with bis vinyl compound $(9 \%)$ and the starting azulene was recovered in a significant amount (39\%) [18]. The considerable stability of thiophenecarbaldehydes in the presence of bases suggested us to synthesize the vinylazulenes substituted at $\mathrm{C}=\mathrm{C}$ bond with 2-thiophenyl or 3-thiophenyl moieties $[19,20]$. More recently, Hawker at al. synthesized and characterized azulene with 2-thiophene moieties at seven membered ring and used these compounds as building blocks for getting stimuli-responsive conjugated oligomers upon exposure to acid [21]. A certain attention has been paid to the behavior of the methyl groups of the seven-membered ring of azulene in the reaction with thiophene-2-carbaldehyde [18]. Even in milder conditions, when sodium $\mathrm{N}$-methylanilide was used as a condensation agent, the yields in alkenes were low due to the small azulene conversion and to other side reactions. The excess of aldehyde produced various ketones as secondary compounds (Scheme 1) [18]. Belfield at al., which described the condensation of guaiazulene 4-methyl group with various aldehydes, in a molten state at $80{ }^{\circ} \mathrm{C}$, reported a yield of $66 \%$ for thiophene-2-carbaldehyde [16]. Therefore, experiments to test the selectivity of the reaction remain necessary when the azulenic substrate or the nature of the aldehydes is varied.

\section{Materials and methods \\ Materials}

Melting points (uncorrected): Kofler apparatus (Reichert, Austria). Elemental analyses: COSTECH Automated elemental analyzer CHNS/halogene by catalytic combustion. UV spectra were 
performed in methanol using a Varian Cary 100 spectrophotometer ( $\lambda$ values are given in $\mathrm{nm}$ and the molar extinction, $\varepsilon$, in $\mathrm{M}^{-1} \mathrm{~cm}^{-1}$ units. ${ }^{1} \mathrm{H}$ - and ${ }^{13} \mathrm{C}-\mathrm{NMR}$ : Bruker Avance DRX4 $\left({ }^{1} \mathrm{H}\right.$ : $400 \mathrm{MHz}$, $\left.{ }^{13} \mathrm{C}: 100.62 \mathrm{MHz}\right)$ for compound characterization and Gemini $300\left({ }^{1} \mathrm{H}: 300 \mathrm{MHz},{ }^{13} \mathrm{C}: 75.47 \mathrm{MHz}\right), J$ values are given in $\mathrm{Hz}$, TMS was used as internal standard in $\mathrm{CDCl}_{3}$ as solvent; signals were assigned on the basis of COSY and HETCOR correlation spectra (the numbering for the compounds was indicated in Schemes). Mass spectra: Varian 310 - MS LC/MS/MS triple quadrupole mass spectrometer fitted with an electrospray ionization interface (ESI). Column chromatography: basic alumina [activity BII-III (Brockmann)] or silica gel [70-230 mesh (ASTM)]. Dichloromethane (DCM) was distilled over calcium hydride and ethyl acetate over anhydrous sodium carbonate.

\section{General Procedure for condensations}

In a $250 \mathrm{~mL}$ two-neck round-bottom flask, equipped with a magnetic stirrer, nitrogen is admitted using an oil trap and azulene derivative $(10 \mathrm{mmol})$ is dissolved in THF $(30 \mathrm{~mL})$, cooled at $-10{ }^{\circ} \mathrm{C}$. Potassium tert-butoxide (3.36g, $30 \mathrm{mmol}$ for each active Me present in the azulene derivative) was added during several minutes in small portions. After another 10-15 min. the corresponding thiophenecarboxaldehyde (30 mmol for each active Me present in azulene) was added using a syringe and then the reaction mixture was let to reach the room temperature. The stirring was continued for $2 \frac{1 / 2}{2}$ hours and then water $(50 \mathrm{~mL})$ was added to quench the butoxide. The monoalkenes can be extracted with petroleum ether but the polyethenes and the ketonic derivatives are extracted from the aqueous layer using methylene chloride until the aqueous layer becomes not color. The organic extracts are washed with water and then mixed, dried on sodium sulfate and evaporated. The residue was chromatographed using silica gel with petroleum ether and then mixture of petroleum ether-methylene chloride to yield the starting azulene, monoalkene, dialkene, trialkene and oxygenated side products. The alkenes are blue or green on column and green as solids.

\section{Vilsmeier formylation}

In a $3 \mathrm{~mL}$ round-bottomed flask equipped with a two-neck adapter, an oil trap for inert inlet, a cork stopper for phosphorous oxychloride admission, 4,6,8-tris(2-thienylvinyl)azulene (98 $\mathrm{mg}, 0.2 \mathrm{mmol}$ ) was dissolved in $0.5 \mathrm{~mL}$ DMF, under an inert atmosphere, and cooled at $0^{\circ} \mathrm{C}$. Then, a solution of $\mathrm{POCl}_{3}$ $(21 \mu \mathrm{L}, 0.23 \mathrm{mmol}$ ) in $0.1 \mathrm{~mL}$ DMF was added dropwise with a syringe during 3-4 min and the reaction mixture was subsequently stirred for $15 \mathrm{~min}$ at this temperature and $30 \mathrm{~min}$. at room temperature. The reaction mixture was cooled back to $0^{\circ} \mathrm{C}$ by adding icy water for the decomposition of $\mathrm{POCl}_{3}$ excess. The resulting solution was then extracted several times with methylene chloride until the aqueous layer has faded (the organic layer is dark green). The combined organic layers were stirred with $\mathrm{NaOH} 20 \%$ for $15 \mathrm{~min}$ in order to hydrolyze completely the intermediate iminium salt. The organic phase is separated and washed with water twice and then dried over anhydrous sodium sulfate. After filtration and solvent evaporation, a chromatographic separation on a silica gel column with methylene chloride as eluent was performed. Three major fraction were obtained, the first one containing the desired product, in a $63 \%$ yield $(64 \mathrm{mg})$.

\section{Wittig reaction}

In a $25 \mathrm{~mL}$ round-bottomed flask equipped with a two-neck adapter, an oil trap for inert inlet, a cork stopper for butyl lithium admission, 2-thienylmethyltriphenylphosphonium chloride (33 mg, 83 $\mu \mathrm{mol})$ is dissolved in diethyl ether $(2 \mathrm{~mL})$ under argon atmosphere, at room temperature. A $15 \%$ solution of BuLi (50-1.32 mg, $75 \mathrm{~mol})$ in hexane was slowly introduced with a syringe, the solution turning orange. After a half hour stirring, 4,6,8-tris((E)-2-(thiophen-2-yl)vinyl)azulene -1-carbaldehyde $26 \mathrm{mg}$ $(50 \mu \mathrm{mol})$ in $3 \mathrm{~mL}$ dioxane was added dropwise, the mixture subsequently refluxed for $3 \mathrm{~h}$ and finally cooled to $0^{\circ} \mathrm{C}$. Icy water was added to quench excess BuLi. The separated organic layers was washed with water, while the reunited aqueous phases were extracted with methylene chloride $(5 \mathrm{~mL})$ until the discoloration of the aqueous phase. The organic layers (dark green) were dried over anhydrous sodium 
sulfate, filtered and the organic solvents distilled. The crude residue was submitted to liquid column chromatography over silica gel, with (9:1 petroleum ether-methylene chloride as eluent. Three green fractions were obtained, the second one containing the desired tetrathienylvinyl derivative, obtained in a $55 \%$ yield $(15 \mathrm{mg}, 26.8 \mu \mathrm{mol})$, which crystalized as orange crystals.

\section{Products characterization}

The compounds are labeled without apostrophe for azulene and with one to four apostrophe for thienyls. The vinyl protons are labeled with Greek signs.

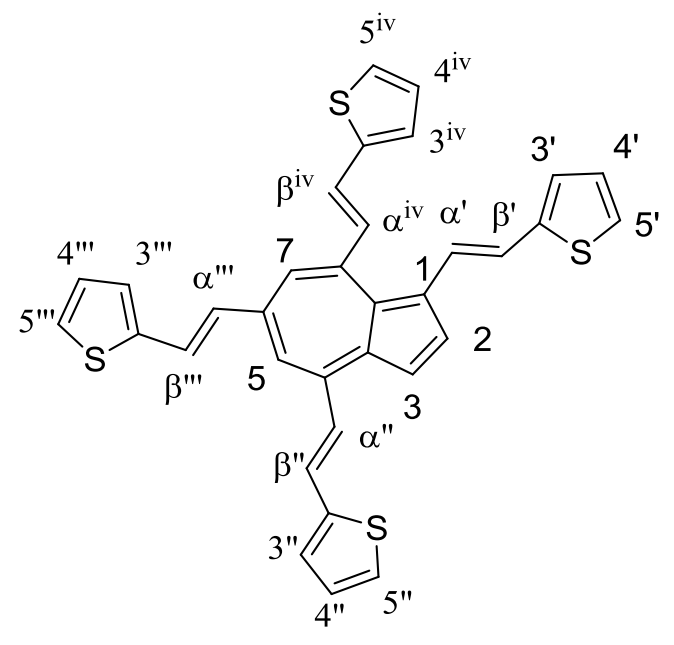

6-[(E)-2-(thien-2-yl)-vinyl]-azulene, 1. Green crystals, m.p. $167^{\circ} \mathrm{C} . \mathrm{UV}-\mathrm{Vis}, \lambda_{\max }(\lg \varepsilon): 202$ (4.41), 274 (3.19), 328 (4.54), 407 (4.54) nm. ${ }^{1} \mathrm{H}-\mathrm{NMR}, \delta$ (ppm): 7.05 (dd, ${ }^{3} \mathrm{~J}=5.0 \mathrm{~Hz},{ }^{3} \mathrm{~J}=3.6 \mathrm{~Hz}, 1 \mathrm{H}, 4$ '-H), $7.11\left(\mathrm{~d},{ }^{3} \mathrm{~J}=15.9 \mathrm{~Hz}, 1 \mathrm{H},=\mathrm{CH}^{\beta}\right), 7.18\left(\mathrm{~d},{ }^{3} \mathrm{~J}=3.6 \mathrm{~Hz}, 1 \mathrm{H}, 3^{\prime}-\mathrm{H}\right), 7.28\left(\mathrm{~d},{ }^{3} \mathrm{~J}=5.0 \mathrm{~Hz}, 1 \mathrm{H}, 5^{\prime}-\mathrm{H}\right), 7.34$ $\left(\mathrm{d},{ }^{3} \mathrm{~J}=3.5 \mathrm{~Hz}, 2 \mathrm{H}, 1-\mathrm{H}, 3-\mathrm{H}\right), 7.35\left(\mathrm{~d},{ }^{3} \mathrm{~J}=10.6 \mathrm{~Hz}, 2 \mathrm{H}, 5-\mathrm{H}, 7-\mathrm{H}\right), 7.46\left(\mathrm{~d},{ }^{3} \mathrm{~J}=16.0 \mathrm{~Hz}, 1 \mathrm{H},=\mathrm{CH}^{\alpha}\right)$, $7.82\left(\mathrm{t},{ }^{3} \mathrm{~J}=3.7 \mathrm{~Hz}, 1 \mathrm{H}, 2-\mathrm{H}\right), 8.28\left(\mathrm{~d},{ }^{3} \mathrm{~J}=10.6 \mathrm{~Hz}, 2 \mathrm{H}, 4-\mathrm{H}, 8-\mathrm{H}\right) .{ }^{13} \mathrm{C}-\mathrm{NMR}, \delta(\mathrm{ppm}): 118.7(\mathrm{C} 1$, C3), 121.5 (C5, C7), 125.5 (C5'), 125.8 (C3'), $127.8\left(\mathrm{C}^{\prime}\right.$ '), $127.8\left(\mathrm{CH}^{\beta}\right), 132.7\left(\mathrm{CH}^{\alpha}, 135.7(\mathrm{C} 2), 136.7\right.$ (C4, C8), 139.2 (C3a, C8a), 142.7 (C2'), 145.7 (C6). MS [ESI]: 237 [M+1]. Calcd. for $\mathrm{C}_{16} \mathrm{H}_{12} \mathrm{~S}: \mathrm{C}$, 81.32; H, 5.12; S, 13.57. Found: C, 81.35, H, 5.15; S, 13.50.

6- $\left[(E)-2-(\right.$ thien-3-yl)-vinyl $]$-azulene, 2 , Green crystals, m.p. $175^{\circ} \mathrm{C} . \mathrm{UV}-\mathrm{Vis}, \lambda_{\max }(\lg \varepsilon): 204$ (4.23), 247 (4.02), 315 (4.52), 392 (4.39) nm. ${ }^{1} \mathrm{H}-\mathrm{NMR}, \delta(\mathrm{ppm}): 7.14\left(\mathrm{~d},{ }^{3} \mathrm{~J}=16.2 \mathrm{~Hz}, 1 \mathrm{H},=\mathrm{CH}^{\alpha}\right), 7.34\left(\mathrm{~d},{ }^{3} \mathrm{~J}\right.$ $=3.7 \mathrm{~Hz}, 2 \mathrm{H}, 1-\mathrm{H}, 3-\mathrm{H}), 7.35\left(\mathrm{~d},{ }^{3} \mathrm{~J}=16.1 \mathrm{~Hz}, 1 \mathrm{H},=\mathrm{CH}^{\alpha}\right), 7.37\left(\mathrm{dd},{ }^{3} \mathrm{~J}=5.1 \mathrm{~Hz},{ }^{4} \mathrm{~J}=0.7 \mathrm{~Hz}, 1 \mathrm{H}, 5^{\prime}-\right.$ H), 7.39 (s, 2 H, 5-H, 7-H), 7.40-7.41 (m, 1 H, 2'-H), 7.41 (dd, $\left.{ }^{3} \mathrm{~J}=4.8 \mathrm{~Hz},{ }^{4} \mathrm{~J}=1.5 \mathrm{~Hz}, 1 \mathrm{H}, 4^{\prime}-\mathrm{H}\right), 7.82$ $\left(\mathrm{t},{ }^{3} \mathrm{~J}=3.6 \mathrm{~Hz}, 1 \mathrm{H}, 2-\mathrm{H}\right), 8.29\left(\mathrm{~d},{ }^{3} \mathrm{~J}=10.6 \mathrm{~Hz}, 2 \mathrm{H}, 4-\mathrm{H}, 8-\mathrm{H}\right) .{ }^{13} \mathrm{C}-\mathrm{NMR}, \delta(\mathrm{ppm}): 119.5(\mathrm{C} 1, \mathrm{C} 3)$, 122.4 (C5, C7), 125.0 (C5'), $126.0\left(\mathrm{C}^{\prime}{ }^{\prime}\right), 127.5\left(\mathrm{C}^{\prime}\right), 127.6\left(\mathrm{CH}^{\beta}\right), 134.1\left(\mathrm{CH}^{\alpha}\right), 136.6(\mathrm{C} 2), 137.5(\mathrm{C} 4$, C8), 140.2 (C3'), 140.3 (C3a, C8a), 147.1 (C6). MS [ESI]: 237 [M+1]. Calcd. for $\mathrm{C}_{16} \mathrm{H}_{12} \mathrm{~S}$ : C, 81.32; H, 5.12; S, 13.57. Found: C, 81.34, H, 5.16; S, 13.50 .

4-[(E)-2-(thien-3-yl)-vinyl]-6-tert-butyl-8-methyl-azulene, 5, Green crystals, m.p. $114{ }^{\circ} \mathrm{C}$. UV-Vis, $\lambda_{\max }$ (lg $\varepsilon$ ): 218 (4.81), 242 (4.32), 270 (4.40), 277 (4.40), 332 (4.66), 388sh (4.27) nm. ${ }^{1} \mathrm{H}-\mathrm{NMR}, \delta$ (ppm): 1.59 (s, $\left.9 \mathrm{H}, \mathrm{Me}_{6}\right), 3.02\left(\mathrm{~s}, 3 \mathrm{H}, \mathrm{Me}_{8}\right), 7.41\left(\mathrm{~d},{ }^{3} \mathrm{~J}=15.6 \mathrm{~Hz}, 1 \mathrm{H},=\mathrm{CH}^{\alpha}\right), 7.43\left(\mathrm{~d},{ }^{3} \mathrm{~J}=3.7 \mathrm{~Hz}, 1\right.$ $\mathrm{H}, 3-\mathrm{H}), 7.45\left(\mathrm{dd},{ }^{3} \mathrm{~J}=4.0 \mathrm{~Hz},{ }^{4} \mathrm{~J}=1.5 \mathrm{~Hz}, 1 \mathrm{H}, 5^{\prime}-\mathrm{H}\right), 7.45\left(\mathrm{~d},{ }^{4} \mathrm{~J}=1.8 \mathrm{~Hz}, 1 \mathrm{H}, 7-\mathrm{H}\right), 7.46\left(\mathrm{~d},{ }^{4} \mathrm{~J}=1.3\right.$ $\left.\mathrm{Hz}, 1 \mathrm{H}, 2^{\prime}-\mathrm{H}\right), 7.56\left(\mathrm{dd},{ }^{3} \mathrm{~J}=5.0 \mathrm{~Hz},{ }^{4} \mathrm{~J}=1.3 \mathrm{~Hz}, 1 \mathrm{H}, 4^{\prime}-\mathrm{H}\right), 7.62\left(\mathrm{~d},{ }^{3} \mathrm{~J}=3.1 \mathrm{~Hz}, 1 \mathrm{H}, 1-\mathrm{H}\right), 7.78\left(\mathrm{~d},{ }^{4} \mathrm{~J}\right.$ $=1.6 \mathrm{~Hz}, 1 \mathrm{H}, 5-\mathrm{H}), 7.80\left(\mathrm{t},{ }^{3} \mathrm{~J}=3.9 \mathrm{~Hz}, 1 \mathrm{H}, 2-\mathrm{H}\right), 8.02\left(\mathrm{~d},{ }^{3} \mathrm{~J}=16 \mathrm{~Hz}, 1 \mathrm{H},=\mathrm{CH}^{\delta}\right) .{ }^{13} \mathrm{C}-\mathrm{NMR}, \delta(\mathrm{ppm})$ : $25.98(\mathrm{Me}), 32.29(\mathrm{MeC}), 39.12(\mathrm{MeC}), 115.0$ (C1), 116.4 (C3), 119.1 (C5), 124.0 (C7), 124.2 (C2'), 125.2 (C4'), $126.6\left(\mathrm{C}^{\prime}\right), 128.1\left(\mathrm{CH}^{\beta}, 131.3\left(\mathrm{CH}^{\alpha}\right), 133.4(\mathrm{C} 2), 135.6(\mathrm{C} 3 \mathrm{a}), 136.9\right.$ (C8a), 140.2 (C3a), 143.2 (C4), 145.2 (C8), 158.0 (C6). MS [ESI]: 307 [M+1]. Calcd. for $\mathrm{C}_{21} \mathrm{H}_{22} \mathrm{~S}: \mathrm{C}, 82.30 ; \mathrm{H}, 7.24 ; \mathrm{S}$, 10.46. Found: C 82.28; H 7.29; S 10.44. 
4,8-bis[(E)-2-(thien-3-yl)-vinyl]-6-tert-butyl-azulene, 7, Green crystals, m.p. $129^{\circ} \mathrm{C}$. UV-Vis, $\lambda_{\max }$ (lg $\varepsilon$ ): 215 (4.38), 244sh (4.25), 289sh (4.33), 336 (4.61), 360sh (4.25) nm. ${ }^{1} \mathrm{H}-\mathrm{NMR}, \delta$ (ppm): 1.61 (s, $18 \mathrm{H}, \mathrm{Me}), 7.39\left(\mathrm{~d},{ }^{3} \mathrm{~J}=16.1 \mathrm{~Hz}, 2 \mathrm{H},=\mathrm{CH}^{\beta}\right), 7.41\left(\mathrm{dd},{ }^{3} \mathrm{~J}=3.0 \mathrm{~Hz},{ }^{4} \mathrm{~J}=1.4 \mathrm{~Hz}, 2 \mathrm{H}, 5^{\prime}-\mathrm{H}\right), 7.46\left(\mathrm{t},{ }^{3} \mathrm{~J}\right.$ $\left.=2.2 \mathrm{~Hz}, 2 \mathrm{H}, 2^{\prime}-\mathrm{H}\right), 7.54\left(\mathrm{dd},{ }^{3} \mathrm{~J}=5.0 \mathrm{~Hz},{ }^{4} \mathrm{~J}=1.5 \mathrm{~Hz}, 2 \mathrm{H}, 4^{\prime}-\mathrm{H}\right), 7.62\left(\mathrm{~d},{ }^{3} \mathrm{~J}=4.0 \mathrm{~Hz}, 2 \mathrm{H}, 1-\mathrm{H}, 3-\mathrm{H}\right)$, $7.77(\mathrm{~s}, 2 \mathrm{H}, 5-\mathrm{H}, 7-\mathrm{H}), 7.79\left(\mathrm{t},{ }^{3} \mathrm{~J}=3.9 \mathrm{~Hz}, 1 \mathrm{H}, 2-\mathrm{H}\right), 8.00\left(\mathrm{~d},{ }^{3} \mathrm{~J}=16.0 \mathrm{~Hz}, 2 \mathrm{H}, \mathrm{CH}^{\alpha}\right) .{ }^{13} \mathrm{C}-\mathrm{NMR}, \delta$ (ppm): $32.25(\mathrm{MeC}), 39.35(\mathrm{MeC}), 115.6(\mathrm{C} 1, \mathrm{C} 3), 119.6(\mathrm{C} 5, \mathrm{C} 7), 123.8\left(\mathrm{C} 2{ }^{\prime}\right), 125.2\left(\mathrm{C}^{\prime}{ }^{\prime}\right), 126.4$ (C5'), $128.0\left(\mathrm{CH}^{\beta}\right)$, $131.3\left(\mathrm{CH}^{\alpha}\right), 133.6(\mathrm{C} 2), 136.0(\mathrm{C} 3 \mathrm{a}, \mathrm{C} 8 \mathrm{a}), 140.2\left(\mathrm{C}^{\prime}\right), 143.2(\mathrm{C} 4, \mathrm{C} 8), 157.7$ (C6). MS [ESI]: 401 [M+1]. Calcd. for $\mathrm{C}_{26} \mathrm{H}_{24} \mathrm{~S}_{2}$ : C, 77.96; H, 6.04; S, 16.00. Found: C, 77.92; H, 6.07; $\mathrm{S}, 16.01$.

6- $\left[(E)-2-(\right.$ thien-3-yl)-vinyl $]-4,8$-dimethyl-azulene, 12, Green crystals, m.p. $106{ }^{\circ} \mathrm{C}$. UV-Vis, $\lambda_{\max }(\lg$

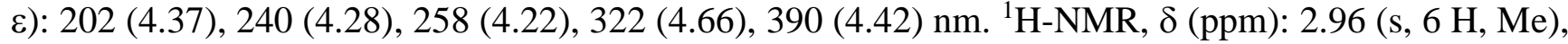
$7.14\left(\mathrm{~d},{ }^{3} \mathrm{~J}=16 \mathrm{~Hz}, 1 \mathrm{H}, \mathrm{CH}^{\beta}\right), 7.33\left(\mathrm{~d},{ }^{3} \mathrm{~J}=16 \mathrm{~Hz}, 1 \mathrm{H}, \mathrm{CH}^{\alpha}\right), 7.35(\mathrm{~s}, 2 \mathrm{H}, 5-\mathrm{H}, 7-\mathrm{H}), 7.36\left(\mathrm{dd},{ }^{3} \mathrm{~J}=5.0\right.$ $\left.\mathrm{Hz},{ }^{4} \mathrm{~J}=3.0 \mathrm{~Hz}, 1 \mathrm{H}, 5^{\prime}-\mathrm{H}\right), 7.38\left(\mathrm{~d},{ }^{3} \mathrm{~J}=3.9 \mathrm{~Hz}, 2 \mathrm{H}, 1-\mathrm{H}, 3-\mathrm{H}\right), 7.38-7.39$ (m, $2 \mathrm{H}, 2$ '-H), 7.42 (dd, ${ }^{3} \mathrm{~J}=$ $4.6 \mathrm{~Hz},{ }^{4} \mathrm{~J}=1.5 \mathrm{~Hz}, 1 \mathrm{H}, 4$ '-H), 7.69 (t, $\left.{ }^{3} \mathrm{~J}=3.9 \mathrm{~Hz}, 1 \mathrm{H}, 2-\mathrm{H}\right) .{ }^{13} \mathrm{C}-\mathrm{NMR}, \delta(\mathrm{ppm}): 25.46(\mathrm{Me}), 116.7$ (C1, C3), 123.8 (C5, C7), $124.4\left(\mathrm{C} 2\right.$ ') $, 125.1\left(\mathrm{C}^{\prime}\right), 126.4\left(\mathrm{C}^{\prime}\right), 126.6\left(\mathrm{CH}^{\beta}\right), 133.6(\mathrm{C} 2), 133.9\left(\mathrm{CH}^{\alpha}\right)$, 136.8, 140.1 (C3'), 143.7 (C4, C8), 145.4 (C6). MS [ESI]: 265 [M+1]. Calcd. for $\mathrm{C}_{18} \mathrm{H}_{16} \mathrm{~S}$ : C, 81.79; H, 6.11; S, 12.11. Found: C, 81.78; H, 6.12; S, 12.11.

4,6-bis[(E)-2-(thien-3-yl)-vinyl]-8-methyl-azulene, 16, Green crystals, m.p. $173{ }^{\circ} \mathrm{C} . \mathrm{UV}-\mathrm{Vis}, \lambda_{\max }(\mathrm{lg}$ ع): 218 (4.81), 242 (4.32), 270 (4.40), 277 (4.40), 332 (4.66), 388sh (4.27) nm. ${ }^{1} \mathrm{H}-\mathrm{NMR}, \delta$ (ppm): 2.96 (s, $3 \mathrm{H}, \mathrm{Me}), 7.18\left(\mathrm{~d},{ }^{3} \mathrm{~J}=16 \mathrm{~Hz}, 1 \mathrm{H},=\mathrm{CH}^{\beta}\right.$ ) $), 7.35\left(\mathrm{~d},{ }^{3} \mathrm{~J}=16 \mathrm{~Hz}, 1 \mathrm{H}, \mathrm{CH}^{\alpha}\right), 7.40\left(\mathrm{~d},{ }^{3} \mathrm{~J}=16 \mathrm{~Hz}, 1 \mathrm{H}\right.$, $\left.\mathrm{CH}^{\beta}{ }^{\prime}\right), 7.40-7.41\left(\mathrm{~m}, 2 \mathrm{H}, 2^{\prime}-\mathrm{H}, 2{ }^{\prime}-\mathrm{H}\right), 7.42\left(\mathrm{~d},{ }^{3} \mathrm{~J}=3.9 \mathrm{~Hz}, 1 \mathrm{H}, 1-\mathrm{H},\right), 7.42\left(\mathrm{~d},{ }^{4} \mathrm{~J}=1.3 \mathrm{~Hz}, 1 \mathrm{H}, 7-\mathrm{H}\right)$, $7.42\left(\mathrm{dd},{ }^{3} \mathrm{~J}=4.1 \mathrm{~Hz},{ }^{4} \mathrm{~J}=1.5 \mathrm{~Hz}, 1 \mathrm{H}, 4{ }^{\prime}{ }^{-}-\mathrm{H}_{6}\right), 7.44\left(\mathrm{dd},{ }^{3} \mathrm{~J}=4.9 \mathrm{~Hz},{ }^{4} \mathrm{~J}=1.5 \mathrm{~Hz}, 1 \mathrm{H}, 4^{\prime}-\mathrm{H}_{4}\right), 7.52$ (dd, $\left.{ }^{3} \mathrm{~J}=5.0 \mathrm{~Hz},{ }^{4} \mathrm{~J}=1.6 \mathrm{~Hz}, 5{ }^{\prime}{ }^{-H_{6}}\right), 7.57\left(\mathrm{dd},{ }^{3} \mathrm{~J}=4.1 \mathrm{~Hz},{ }^{4} \mathrm{~J}=1.5 \mathrm{~Hz}, 1 \mathrm{H}, 5^{\prime}-\mathrm{H}_{4}\right), 7.57\left(\mathrm{~d},{ }^{3} \mathrm{~J}=3.9 \mathrm{~Hz}, 1\right.$ $\mathrm{H}, 3-\mathrm{H}), 7.67\left(\mathrm{~d},{ }^{4} \mathrm{~J}=1.3 \mathrm{~Hz}, 1 \mathrm{H}, 5-\mathrm{H}\right), 7.73\left(\mathrm{t},{ }^{3} \mathrm{~J}=3.9 \mathrm{~Hz}, 1 \mathrm{H}, 2-\mathrm{H}\right), 7.93\left(\mathrm{~d},{ }^{3} \mathrm{~J}=16 \mathrm{~Hz}, 1 \mathrm{H}, \mathrm{CH}^{\alpha}\right)$. ${ }^{13} \mathrm{C}-\mathrm{NMR}, \delta(\mathrm{ppm}): 25.63(\mathrm{Me}), 115.9$ (C1), 117.4 (C3), 120.2 (C7), 124.0 (C2”), 124.2 (C2'), 125.1 (C4”), 125.2 (C4'), 126.3 ( $\mathrm{CH}^{\beta}, 126.6$ (C5', C5”), $128.5\left(\mathrm{CH}^{\alpha}\right), 130.5\left(\mathrm{CH}^{\alpha}\right), 133.7(\mathrm{C} 2), 134.1\left(\mathrm{CH}^{\alpha}\right)$, 135.9 (C3a), 137.1 (C8a), 140.0 (C3”), 140.2 (C3'), 143.4 (C8), 143.5 (C4), 145.3 (C6). MS [ESI]: 359 [M+1]. Calcd. for $\mathrm{C}_{23} \mathrm{H}_{18} \mathrm{~S}_{2}$ : C, 77.08; H, 5.07; S, 17.85. Found: C, 77.07; H, 5.10; S, 17.83.

4,6,8-tris [(E)-2-(thien-3-yl)-vinyl]-azulene, 18, Green crystals, m.p. $168^{\circ} \mathrm{C}$. UV-Vis, $\lambda_{\max }(\lg \varepsilon): 218$ (4.81), 256 (4.48), 263 (4.74), 270 (4.49), 277 (4.50), 290 (4.44), 350 (4.83), 400sh (4.43) nm. ${ }^{1} \mathrm{H}-\mathrm{NMR}$, $\delta(\mathrm{ppm}): 7.24\left(\mathrm{~d},{ }^{3} \mathrm{~J}=16 \mathrm{~Hz}, 1 \mathrm{H},=\mathrm{CH}^{\beta}{ }^{\prime}\right), 7.37\left(\mathrm{~d},{ }^{3} \mathrm{~J}=15.6 \mathrm{~Hz}, 1 \mathrm{H},=\mathrm{CH}^{\alpha}\right), 7.38\left(\mathrm{dd},{ }^{3} \mathrm{~J}=5.1 \mathrm{~Hz},{ }^{4} \mathrm{~J}\right.$ $=2.9 \mathrm{~Hz}, 1 \mathrm{H}, 5^{\prime}$ '-H), $7.41\left(\mathrm{ddd},{ }^{3} \mathrm{~J}=5.1 \mathrm{~Hz},{ }^{4} \mathrm{~J}=2.9 \mathrm{~Hz},{ }^{4} \mathrm{~J}=0.6 \mathrm{~Hz}, 2 \mathrm{H}, 5^{\prime}-\mathrm{H}\right), 7.41\left(\mathrm{~d},{ }^{3} \mathrm{~J}=16 \mathrm{~Hz}, 2\right.$ $\left.\mathrm{H},=\mathrm{CH}^{\beta}{ }^{\prime}\right), 7.42\left(\mathrm{ddd},{ }^{4} \mathrm{~J}=2.4 \mathrm{~Hz},{ }^{4} \mathrm{~J}=1.2 \mathrm{~Hz}, 1 \mathrm{H}, 2\right.$ ' $\left.-\mathrm{H}\right), 7.45\left(\mathrm{dd}, 2 \mathrm{H},{ }^{4} \mathrm{~J}=2.3 \mathrm{~Hz},{ }^{4} \mathrm{~J}=1.3 \mathrm{~Hz}, 2^{\prime}-\right.$ $\mathrm{H}), 7.46\left(\mathrm{dd},{ }^{3} \mathrm{~J}=4.9 \mathrm{~Hz},{ }^{4} \mathrm{~J}=1.0 \mathrm{~Hz}, 1 \mathrm{H}, 4{ }^{\prime}{ }^{-H}\right), 7.51\left(\mathrm{dd},{ }^{3} \mathrm{~J}=5.0 \mathrm{~Hz},{ }^{4} \mathrm{~J}=1.3 \mathrm{~Hz}, 2 \mathrm{H}, 4{ }^{\prime}-\mathrm{H}\right), 7.58(\mathrm{~d}$, $\left.{ }^{3} \mathrm{~J}=4.1 \mathrm{~Hz}, 2 \mathrm{H}, 1-\mathrm{H}, 3-\mathrm{H}\right), 7.68(\mathrm{~s}, 2 \mathrm{H}, 5-\mathrm{H}, 7-\mathrm{H}), 7.72\left(\mathrm{t},{ }^{3} \mathrm{~J}=3.9 \mathrm{~Hz}, 1 \mathrm{H}, 2-\mathrm{H}\right), 7.91\left(\mathrm{~d},{ }^{3} \mathrm{~J}=16 \mathrm{~Hz}\right.$, $2 \mathrm{H},=\mathrm{CH}^{\alpha}$ ). ${ }^{13} \mathrm{C}-\mathrm{NMR}, \delta(\mathrm{ppm}): 116.6(\mathrm{C} 1, \mathrm{C} 3), 120.0$ (C5, C7), 124.1 (C2”), 124.2 (C2'), 125.0 (C4”), 125.2 (C4'), $126.4\left(\mathrm{CH}^{\beta^{\prime}}\right), 126.7$ (C5',C5”), $128.5\left(\mathrm{CH}^{\beta^{\prime}}\right), 130.6\left(\mathrm{CH}^{\alpha}\right), 133.9(\mathrm{C} 2), 134.3\left(\mathrm{CH}^{\square}\right), 136.2$ (C3a, C8a), 139.9 (C3”), 140.1 (C3'), 143.4 (C4, C8), 143.6 (C6). MS [ESI]: 453 [M+1]. Calcd. for $\mathrm{C}_{28} \mathrm{H}_{20} \mathrm{~S}_{3}$ : C, 74.32; H, 4.46; S, 21.22. Found: C, 74.35; H, 4.48; S, 21.17.

4,6,8-tris[(E)-2-(thien-2-yl)-vinyl]-azulene, 17, Green crystals, m.p. $180^{\circ} \mathrm{C}$. UV-Vis, $\lambda_{\max }(\lg \varepsilon): 204$ (4.11), 238 (4.01), 283 (4.15), 285 (4.15), 365 (4.51), 368 (4.51), 415sh (4.27) nm. ${ }^{1} \mathrm{H}-\mathrm{NMR}, \delta$ (ppm): $7.05\left(\mathrm{dd},{ }^{3} \mathrm{~J}=5.0 \mathrm{~Hz},{ }^{4} \mathrm{~J}=3.6 \mathrm{~Hz}, 1 \mathrm{H}, 4\right.$ "'-H), $7.09\left(\mathrm{dd},{ }^{3} \mathrm{~J}=5.0 \mathrm{~Hz},{ }^{4} \mathrm{~J}=3.6 \mathrm{~Hz}, 2 \mathrm{H}, 4^{\prime}-\mathrm{H}\right), 7.19$ (d, ${ }^{3} \mathrm{~J}$ $=16.2 \mathrm{~Hz}, 1 \mathrm{H}, \mathrm{CH}^{\beta}$ ”), $7.23\left(\mathrm{~d},{ }^{3} \mathrm{~J}=3.6 \mathrm{~Hz}, 1 \mathrm{H}, 3\right.$ "'-H), $7.24\left(\mathrm{~d},{ }^{3} \mathrm{~J}=3.6 \mathrm{~Hz}, 1 \mathrm{H}, 3^{\prime}-\mathrm{H}\right), 7.30\left(\mathrm{~d},{ }^{3} \mathrm{~J}=5.2\right.$ $\left.\mathrm{Hz}, 1 \mathrm{H}, 5^{\prime \prime}-\mathrm{H}\right), 7.33$ (d, $\left.{ }^{3} \mathrm{~J}=5.0 \mathrm{~Hz}, 2 \mathrm{H}, 5^{\prime}-\mathrm{H}\right), 7.48\left(\mathrm{~d},{ }^{3} \mathrm{~J}=15.9 \mathrm{~Hz}, 1 \mathrm{H} \mathrm{CH}^{\alpha}\right.$ ) $) 7.52\left(\mathrm{~d},{ }^{3} \mathrm{~J}=15.9 \mathrm{~Hz}\right.$, $2 \mathrm{H} \mathrm{CH}^{\beta}$ ), $7.59\left(\mathrm{~d},{ }^{3} \mathrm{~J}=4.0 \mathrm{~Hz}, 2 \mathrm{H}, 1-\mathrm{H}, 3-\mathrm{H}\right), 7.62(\mathrm{~s}, 2 \mathrm{H}, 5-\mathrm{H}, 7-\mathrm{H}), 7.73\left(\mathrm{t},{ }^{3} \mathrm{~J}=4.0 \mathrm{~Hz}, 1 \mathrm{H}, 2-\mathrm{H}\right)$, $7.87\left(\mathrm{~d},{ }^{3} \mathrm{~J}=15.8 \mathrm{~Hz}, 2 \mathrm{H} \mathrm{CH}^{\alpha}\right) .{ }^{13} \mathrm{C}-\mathrm{NMR}, \delta(\mathrm{ppm}): 116.9$ (C1, C3), 119.7 (C5, C7), 125.3 (C5"), 125.6 (C5', C3”), 125.8 (C3', CH"), 127.5 (CH'), 127.7 (C4”), 128.0 (C4'), $130.1\left(\mathrm{CH}^{\alpha}\right), 133.8(\mathrm{C} 2), 134.2$ 
$\left(\mathrm{CH}^{\alpha ”}\right), 136.3$ (C3a, C8a), 142.6 (C2”), 142.8 (C2', C6), 142.9 (C4, C8). MS [ESI]: 453 [M+1]. Calcd. for $\mathrm{C}_{28} \mathrm{H}_{20} \mathrm{~S}_{3}$ : C, 74.32; H, 4.46; S, 21.22. Found: C, 74.30; H, 4.47; S, 21.23.

4-[(E)-2-(thien-2-yl)-vinyl-6,8-bis(thien-2-ylcarbonylmethylene)-azulene, 20, Green oil, ${ }^{1} \mathrm{H}-\mathrm{NMR}, \delta$ (ppm): 4.25 (s, $\left.2 \mathrm{H}, \mathrm{CH}_{2}{ }^{6}\right), 4.64$ (s, $\left.2 \mathrm{H}, \mathrm{CH}_{2}{ }^{8}\right), 7.01-7.04$ (m, $3 \mathrm{H}, 4^{\prime}-\mathrm{H}, 4$ "'-H, 4"'-H), 7.12 (s, $1 \mathrm{H}, 7-$ $\mathrm{H}), 7.14\left(\mathrm{~d},{ }^{3} \mathrm{~J}=3.5 \mathrm{~Hz}, 1 \mathrm{H}, 3^{\prime}-\mathrm{H}\right), 7.27\left(\mathrm{~d},{ }^{3} \mathrm{~J}=5.1 \mathrm{~Hz}, 1 \mathrm{H}, 5^{\prime}-\mathrm{H}\right), 7.40\left(\mathrm{~d},{ }^{3} \mathrm{~J}=3.9 \mathrm{~Hz}, 1 \mathrm{H}, 1-\mathrm{H}\right), 7.44$ $\left(\mathrm{d},{ }^{3} \mathrm{~J}=15.8 \mathrm{~Hz}, 1 \mathrm{H},=\mathrm{CH}^{\beta}\right.$ ) $, 7.48\left(\mathrm{~d},{ }^{3} \mathrm{~J}=1.2 \mathrm{~Hz}, 1 \mathrm{H}, 5-\mathrm{H}\right), 7.55-7.57$ (m, $2 \mathrm{H}, 5$ '-H, 5-H'”), 7.61 (dd, $\left.{ }^{3} \mathrm{~J}=4.1 \mathrm{~Hz},{ }^{4} \mathrm{~J}=1.5 \mathrm{~Hz}, 1 \mathrm{H}, 3-\mathrm{H}\right), 7.69\left(\mathrm{~d},{ }^{3} \mathrm{~J}=3.9 \mathrm{~Hz}, 1 \mathrm{H}, 3{ }^{\prime}-\mathrm{H}\right), 7.73\left(\mathrm{~d},{ }^{3} \mathrm{~J}=3.9 \mathrm{~Hz}, 1 \mathrm{H}, 3{ }^{\prime \prime}{ }_{-} \mathrm{H}\right)$, $7.74\left(\mathrm{t},{ }^{3} \mathrm{~J}=4.0 \mathrm{~Hz}, 1 \mathrm{H}, 2-\mathrm{H}\right), 7.81\left(\mathrm{~d},{ }^{3} \mathrm{~J}=16.0 \mathrm{~Hz}, 1 \mathrm{H},=\mathrm{CH}^{\alpha}\right) .{ }^{13} \mathrm{C}-\mathrm{NMR} \delta(\mathrm{ppm}): 48.91\left(\mathrm{CH}_{2}{ }^{4}\right)$, $52.23\left(\mathrm{CH}_{2}{ }^{6}\right), 116.5(\mathrm{C} 1), 117.6(\mathrm{C} 3), 123.1\left(\mathrm{C}^{\prime}\right), 125.9\left(\mathrm{C}^{\prime}\right), 127.9\left(\mathrm{CH}_{\alpha}\right), 128.0\left(\mathrm{C}^{\prime}\right), 128.1(\mathrm{C} 5$, C7), 128.3 (C4"'), 128.4 (C4"), 132.6 (C5"'), 133.0 (C5"), 134.1 (C3"”), 134.3 (C3"), 135.0 (C2), 136.4 (C3a), 137.4 (C2'), 141.2 (C2"”, C2”), 142.5 (C4), 142.9 (C8), 143.6 (C8a), 143.8 (C6), 189.8 (CO'), $190.0\left(\mathrm{CO}^{6}\right)$. MS [ESI]: 485 [M+1]. Calcd. for $\mathrm{C}_{28} \mathrm{H}_{20} \mathrm{O}_{2} \mathrm{~S}_{3}: \mathrm{C}, 69.39 ; \mathrm{H}, 4.16 ; \mathrm{O}, 6.60 ; \mathrm{S}, 19.85$. Found: C, 69.32; H, 4.20; S, 19.75 .

4-(Thien-2-ylcarbonylmethylene)-6-[(E)-2-(thien-2-yl)-vinyl]-8-methylazulene, 19, Green oil, ${ }^{1} \mathrm{H}-$ NMR, $\delta(\mathrm{ppm}): 2.89\left(\mathrm{~s}, 3 \mathrm{H}, \mathrm{Me}^{8}\right), 4.74\left(\mathrm{~s}, 2 \mathrm{H}, \mathrm{CH}_{2}{ }^{4}\right), 7.02\left(\mathrm{dd},{ }^{3} \mathrm{~J}=5.0 \mathrm{~Hz},{ }^{3} \mathrm{~J}=3.5 \mathrm{~Hz}, 1 \mathrm{H}, 4^{\prime}-\mathrm{H}\right)$, $7.03\left(\mathrm{~d},{ }^{3} \mathrm{~J}=16.0 \mathrm{~Hz}, 1 \mathrm{H}, \mathrm{CH}^{\beta}\right.$ ) $), 7.07\left(\mathrm{dd},{ }^{3} \mathrm{~J}=5.1 \mathrm{~Hz},{ }^{3} \mathrm{~J}=3.9 \mathrm{~Hz}, 1 \mathrm{H}, 4{ }^{\prime}-\mathrm{H}\right), 7.17\left(\mathrm{~d},{ }^{3} \mathrm{~J}=3.9 \mathrm{~Hz}, 1\right.$ H, 3'-H), $7.25\left(\mathrm{dd},{ }^{3} \mathrm{~J}=5.1 \mathrm{~Hz},{ }^{4} \mathrm{~J}=1.2 \mathrm{~Hz}, 5^{\prime}-\mathrm{H}\right), 7.29(\mathrm{~s}, 1 \mathrm{H}, 7-\mathrm{H}), 7.31(\mathrm{~s}, 1 \mathrm{H}, 5-\mathrm{H}), 7.33\left(\mathrm{~d},{ }^{3} \mathrm{~J}=\right.$ $\left.16.0 \mathrm{~Hz}, 1 \mathrm{H}, \mathrm{CH}^{\alpha}\right), 7.36\left(\mathrm{~d},{ }^{3} \mathrm{~J}=3.9 \mathrm{~Hz}, 1 \mathrm{H}, 1-\mathrm{H}\right), 7.38\left(\mathrm{~d},{ }^{3} \mathrm{~J}=3.9 \mathrm{~Hz}, 1 \mathrm{H}, 3-\mathrm{H}\right), 7.61\left(\mathrm{dd},{ }^{3} \mathrm{~J}=5.1\right.$ $\mathrm{Hz},{ }^{4} \mathrm{~J}=1.1 \mathrm{~Hz}, 5$ "'-H), $7.68\left(\mathrm{t},{ }^{3} \mathrm{~J}=3.9 \mathrm{~Hz}, 1 \mathrm{H}, 2-\mathrm{H}\right), 7.81\left(\mathrm{dd},{ }^{3} \mathrm{~J}=3.5 \mathrm{~Hz},{ }^{4} \mathrm{~J}=1,1 \mathrm{~Hz}, 1 \mathrm{H}, 3\right.$ "'-H). ${ }^{13} \mathrm{C}-\mathrm{NMR}, \delta(\mathrm{ppm}): 25.39(\mathrm{Me}), 48.91\left(\mathrm{CH}_{2}\right), 116.5(\mathrm{C} 1), 117.3(\mathrm{C} 3), 124.4(\mathrm{C} 7), 124.8$ (C5), 125.4 (C5”), 125.6 (C3”), 127.6 (C4”), 127.9 (CH $\left.{ }^{\beta}\right), 128.3$ (C4'), 132.5 (C5'), $132.9\left(\mathrm{CH}^{\square}\right), 134.1$ (C3'), 134.5 (C2), 136.9 (C3a), 137.2 (C8a), 140.8 (C2”), 142.5 (C8), 143.1 (C2'), 143.9 (C4), 145.5 (C6), 189.9 (CO). MS [ESI]: 377 [M+1]. Calcd. for $\mathrm{C}_{23} \mathrm{H}_{20} \mathrm{OS}_{2}$ : C, 73.39; H, 5.36; S, 17.00. Found: C, 73.32; H, 5.39; S, 17.05 .

(8-Methyl-6-tert-butylazulen-4-yl)-1-(thien-3-yl)ethanone, 9, Lila oil, ${ }^{1} \mathrm{H}-\mathrm{NMR}, \delta$ (ppm): 1.50 (s, 9 $\left.\mathrm{H}, \mathrm{Me}_{3} \mathrm{C}\right), 3.01\left(\mathrm{~s}, 3 \mathrm{H}, \mathrm{Me}_{8}\right), 4.81\left(\mathrm{~s}, 2 \mathrm{H}, \mathrm{CH}_{2}\right), 7.28\left(\mathrm{ddd},{ }^{3} \mathrm{~J}=5.1 \mathrm{~Hz},{ }^{4} \mathrm{~J}=2.9 \mathrm{~Hz},{ }^{4} \mathrm{~J}=1.0 \mathrm{~Hz}, 1 \mathrm{H}\right.$, 4'-H), $7.41(\mathrm{~s}, 1 \mathrm{H}, 7-\mathrm{H}), 7.42(\mathrm{~s}, 1 \mathrm{H}, 5-\mathrm{H}), 7.43\left(\mathrm{dd},{ }^{3} \mathrm{~J}=3.8 \mathrm{~Hz}, 1 \mathrm{H}, 1-\mathrm{H}\right), 7.45\left(\mathrm{dd},{ }^{3} \mathrm{~J}=3.9 \mathrm{~Hz}, 1 \mathrm{H}\right.$, 3-H), $7.64\left(\mathrm{ddd},{ }^{3} \mathrm{~J}=5.1 \mathrm{~Hz},{ }^{4} \mathrm{~J}=2.8 \mathrm{~Hz},{ }^{4} \mathrm{~J}=1.1 \mathrm{~Hz}, 1 \mathrm{H}, 5^{\prime}-\mathrm{H}\right), 7.79\left(\mathrm{t},{ }^{3} \mathrm{~J}=4.0 \mathrm{~Hz}, 1 \mathrm{H}, 2-\mathrm{H}\right), 8.16$ $\left(\mathrm{dd},{ }^{4} \mathrm{~J}=2.9 \mathrm{~Hz},{ }^{4} \mathrm{~J}=1.0 \mathrm{~Hz}, 1 \mathrm{H}, 2^{\prime}-\mathrm{H}\right) .{ }^{13} \mathrm{C}-\mathrm{NMR}, \delta(\mathrm{ppm}): 25.90\left(\mathrm{Me}_{8}\right), 32.09\left(\mathrm{Me}_{6}\right), 38.83\left(\mathrm{C}_{6}\right), 49.85$ $\left(\mathrm{CH}_{2}\right), 115.5$ (C1), 116.4 (C3), 124.0 (C7), 124.5 (C5), 126.4 (C4'), 127.3 (C5'), 132.6 (C2'), 134.1 (C2), 136.6 (C3a), 137.0 (C8a), 141.2 (C3'), 142.0 (C4), 145.6 (C8), 158.3 (C6), 191.7 (CO). MS [ESI]: 325 [M+1]. Calcd. for $\mathrm{C}_{21} \mathrm{H}_{24} \mathrm{OS}$ : C, 77.73; H, 7.46; O, 4.93; S, 9.88. Found: C, 77.65; H, 7.49; S, 9.86. 2-\{8-Methyl-6-[(E)-2-(thien-3-yl)-vinyl]-azulen-4-yl\}-1-(thien-3-yl)-ethanone, 21, Green oil, ${ }^{1} \mathrm{H}-$ NMR, $\delta$ (ppm): 2.94 (s, $3 \mathrm{H}, \mathrm{Me}), 4.76\left(\mathrm{~s}, 2 \mathrm{H}, \mathrm{CH}_{2}\right), 7.08\left(\mathrm{~d},{ }^{3} \mathrm{~J}=16.2 \mathrm{~Hz}, 1 \mathrm{H},=\mathrm{CH}^{\beta}\right.$ ), 7.29 (ddd, ${ }^{3} \mathrm{~J}$ $\left.=5.1 \mathrm{~Hz},{ }^{4} \mathrm{~J}=2.9 \mathrm{~Hz}, 1 \mathrm{H}, 4^{\prime}-\mathrm{H}\right), 7.37\left(\mathrm{~d},{ }^{3} \mathrm{~J}=5.1 \mathrm{~Hz}, 1 \mathrm{H}, 5^{\prime}{ }^{-}-\mathrm{H}\right), 7.39\left(\mathrm{~d},{ }^{3} \mathrm{~J}=16.0 \mathrm{~Hz}, 1 \mathrm{H},=\mathrm{CH}^{\beta^{\prime}}\right)$, 7.40-7.41 (m, $1 \mathrm{H}, 2$ '-H), 7.41 (bd, ${ }^{3} \mathrm{~J}=4.8 \mathrm{~Hz}, 1 \mathrm{H}, 4$ "'-H), 7.43 (dd, $\left.{ }^{3} \mathrm{~J}=3.8 \mathrm{~Hz}, 1 \mathrm{H}, 1-\mathrm{H}\right), 7.45$ (dd, $\left.{ }^{3} \mathrm{~J}=3.9 \mathrm{~Hz}, 1 \mathrm{H}, 3-\mathrm{H}\right), 7.62\left(\mathrm{dd},{ }^{3} \mathrm{~J}=5.1 \mathrm{~Hz},{ }^{4} \mathrm{~J}=1.1 \mathrm{~Hz}, 5^{\prime}-\mathrm{H}\right), 7.71\left(\mathrm{t},{ }^{3} \mathrm{~J}=3.9 \mathrm{~Hz}, 1 \mathrm{H}, 2-\mathrm{H}\right), 8.19(\mathrm{dd}$, $\left.{ }^{4} \mathrm{~J}=2.9 \mathrm{~Hz}, 1 \mathrm{H}, 2^{\prime}-\mathrm{H}\right)$. MS [ESI]: 377 [M+1]. Calcd. for $\mathrm{C}_{23} \mathrm{H}_{20} \mathrm{OS}_{2}$ : C, 73.37; H, 5.35; S, 17.03. Found: C, 73.40; H, 5.38; S, 17.05 .

4,6,8-tris((E)-2-(thiophen-2-yl)vinyl)azulene-1-carbaldehyde, 17a, Green crystals, m.p. $195{ }^{\circ} \mathrm{C} .{ }^{1} \mathrm{H}-$ NMR, $\delta(\mathrm{ppm}): 7.04\left(\mathrm{~d},{ }^{3} \mathrm{~J}=16.2 \mathrm{~Hz}, 1 \mathrm{H}, \mathrm{CH}^{\beta}\right.$ ), $7.06\left(\mathrm{dd},{ }^{3} \mathrm{~J}=5.0 \mathrm{~Hz},{ }^{4} \mathrm{~J}=3.6 \mathrm{~Hz}, 2 \mathrm{H}, 4-\mathrm{H}^{\prime}\right), 7.08$ $\left(\mathrm{dd},{ }^{3} \mathrm{~J}=5.0 \mathrm{~Hz},{ }^{4} \mathrm{~J}=3.6 \mathrm{~Hz}, 1 \mathrm{H}, 4-\mathrm{H}^{\prime \prime}\right), 7.20-7.24$ (m, $\left.3 \mathrm{H}, 3-\mathrm{H}^{\prime}, 3-\mathrm{H}^{\prime}\right), 7.32$ (d, ${ }^{3} \mathrm{~J}=5.0 \mathrm{~Hz}, 1 \mathrm{H}, 5-$ $\mathrm{H}^{\prime}$ ), $7.33\left(\mathrm{~d},{ }^{3} \mathrm{~J}=15.8 \mathrm{~Hz}, 1 \mathrm{H}, \mathrm{CH}^{\beta^{\prime}}\right), 7.34\left(\mathrm{~d},{ }^{3} \mathrm{~J}=5.7 \mathrm{~Hz}, 1 \mathrm{H}, 5-\mathrm{H}^{\prime}\right), 7.35\left(\mathrm{~d},{ }^{3} \mathrm{~J}=4.0 \mathrm{~Hz}, 1 \mathrm{H}, 3-\mathrm{H}\right)$, $7.39\left(\mathrm{~d},{ }^{3} \mathrm{~J}=4.8 \mathrm{~Hz}, 1 \mathrm{H}, 5-\mathrm{H}^{\prime \prime}\right), 7.40\left(\mathrm{~d},{ }^{3} \mathrm{~J}=15.9 \mathrm{~Hz}, 1 \mathrm{H}=\mathrm{CH}^{\beta^{\prime \prime}}\right), 7.44\left(\mathrm{~d},{ }^{3} \mathrm{~J}=15.8 \mathrm{~Hz}, 1 \mathrm{H}=\mathrm{CH}^{\alpha}\right)$, $7.67\left(\mathrm{~d},{ }^{3} \mathrm{~J}=15.6 \mathrm{~Hz}, 1 \mathrm{H} \mathrm{CH}^{\alpha}\right), 7.68(\mathrm{~s}, 2 \mathrm{H}, 5-\mathrm{H}, 7-\mathrm{H}), 7.70\left(\mathrm{~d},{ }^{3} \mathrm{~J}=15.7 \mathrm{~Hz}, 1 \mathrm{H} \mathrm{CH}^{\alpha}{ }^{\prime \prime}\right), 8.18\left(\mathrm{t},{ }^{3} \mathrm{~J}=\right.$ $4.6 \mathrm{~Hz}, 1 \mathrm{H}, 2-\mathrm{H}), 10.52$ (s, 1H, CHO). ${ }^{13} \mathrm{C}-\mathrm{NMR}, \delta$ (ppm): 117.3 (C3), 124.4 (C7), 125.8 (C5), 126.5 (C5”), 126.6 (C5'), 127.1 (C3”), 127.5 (C3'), 128.0 (CH”), 128.1 (C4”), 128.5 (C4'), 128.9 (CH $\left.{ }^{\beta}\right)$, 130.6q(C1), $131.9\left(\mathrm{CH}^{\alpha^{\prime}}\right), 133.3\left(\mathrm{CH}^{\alpha}\right), 136.7 \mathrm{q}(\mathrm{C} 3 \mathrm{a}), 137.2(\mathrm{C} 2), 141.7(\mathrm{C} 8), 141.8 \mathrm{q}(\mathrm{C} 8 \mathrm{a}), 141.9 \mathrm{q}$ 
(C2”), 142.0q (C2'), 144.2q (C4), 145.4q (C6), 186.6 (CHO). MS [ESI]: 561 [M+1]. Calcd. for $\mathrm{C}_{34} \mathrm{H}_{24} \mathrm{~S}_{4}: \mathrm{C}, 72.82 ; \mathrm{H}, 4.31 ; \mathrm{S}, 22.87$. Found: C, 72.81; H, 4.32; S, 22.87.

1,4,6,8-tetrakis((E)-2-(thiophen-2-yl)vinyl)azulene, 22, Dark green crytals, m.p. $227{ }^{\circ} \mathrm{C}$. UV-Vis, $\lambda_{\max }(\lg \varepsilon): 201$ (4.36), 221 (4.25), 279 (4.14), 418 (4.58) nm. ${ }^{1} \mathrm{H}-\mathrm{NMR}, \delta(\mathrm{ppm}): 6.92$ (dd, ${ }^{3} \mathrm{~J}=5.0 \mathrm{~Hz}$, $\left.{ }^{4} \mathrm{~J}=3.6 \mathrm{~Hz}, 1 \mathrm{H}, 4-\mathrm{H}^{\prime}\right), 6.97\left(\mathrm{~d},{ }^{3} \mathrm{~J}=3.6 \mathrm{~Hz}, 1 \mathrm{H}, 3-\mathrm{H}^{\prime}\right), 7.06\left(\mathrm{~d},{ }^{3} \mathrm{~J}=16.2 \mathrm{~Hz}, 1 \mathrm{H}, \mathrm{CH}^{\beta}\right), 7.07\left(\mathrm{~d},{ }^{3} \mathrm{~J}=\right.$ $\left.5.2 \mathrm{~Hz}, 1 \mathrm{H}, 4-\mathrm{H}^{\prime}\right), 7.40$ (d, ${ }^{3} \mathrm{~J}=16.2 \mathrm{~Hz}, 1 \mathrm{H} \mathrm{CH}^{\beta}$ ), $7.02\left(\mathrm{dd},{ }^{3} \mathrm{~J}=5.0 \mathrm{~Hz},{ }^{4} \mathrm{~J}=3.6 \mathrm{~Hz}, 1 \mathrm{H}, 4-\mathrm{H}^{\prime \prime}\right), 7.04$ $\left(\mathrm{dd},{ }^{3} \mathrm{~J}=5.0 \mathrm{~Hz},{ }^{4} \mathrm{~J}=3.6 \mathrm{~Hz}, 2 \mathrm{H}, 4-\mathrm{H}\right.$ ", $\left.4-\mathrm{H}^{\mathrm{iv}}\right), 7.08\left(\mathrm{~d},{ }^{3} \mathrm{~J}=16.2 \mathrm{~Hz}, 1 \mathrm{H}, \mathrm{CH}^{\beta^{\prime} ”}\right), 7.20\left(\mathrm{~d},{ }^{3} \mathrm{~J}=3.6 \mathrm{~Hz}\right.$, $\left.1 \mathrm{H}, 3-\mathrm{H}^{\prime \prime}\right), 7.22\left(\mathrm{~d},{ }^{3} \mathrm{~J}=3.6 \mathrm{~Hz}, 1 \mathrm{H}, 3-\mathrm{H}^{\prime}\right), 7.24\left(\mathrm{~d},{ }^{3} \mathrm{~J}=3.6 \mathrm{~Hz}, 1 \mathrm{H}, 3-\mathrm{H}^{\mathrm{iv}}\right), 7.27\left(\mathrm{~d},{ }^{3} \mathrm{~J}=5.2 \mathrm{~Hz}, 1 \mathrm{H}\right.$, $\left.5-\mathrm{H}^{\prime \prime}\right), 7.29$ (d, $\left.{ }^{3} \mathrm{~J}=5.2 \mathrm{~Hz}, 1 \mathrm{H}, 5-\mathrm{H}^{\prime}\right), 7.31\left(\mathrm{~d},{ }^{3} \mathrm{~J}=5.0 \mathrm{~Hz}, 1 \mathrm{H}, 5-\mathrm{H}^{\mathrm{iv}}\right), 7.69\left(\mathrm{~d},{ }^{3} \mathrm{~J}=15.9 \mathrm{~Hz}, 1 \mathrm{H}\right.$ $\left.\mathrm{CH}^{\alpha " ”}\right), 7.72\left(\mathrm{~d},{ }^{3} \mathrm{~J}=15.9 \mathrm{~Hz}, 1 \mathrm{H} \mathrm{CH}^{\alpha}\right.$ ), $7.76\left(\mathrm{~d},{ }^{3} \mathrm{~J}=15.9 \mathrm{~Hz}, 2 \mathrm{H} \mathrm{CH}^{\alpha \mathrm{iv}}\right), 7.48\left(\mathrm{~d},{ }^{3} \mathrm{~J}=4.0 \mathrm{~Hz}, 1 \mathrm{H}, 3-\right.$ $\mathrm{H}), 7.42(\mathrm{~s}, 1 \mathrm{H}, 5-\mathrm{H}), 7.43(\mathrm{~s}, 1 \mathrm{H}, 7-\mathrm{H}), 7.88\left(\mathrm{t},{ }^{3} \mathrm{~J}=4.0 \mathrm{~Hz}, 1 \mathrm{H}, 2-\mathrm{H}\right) .{ }^{13} \mathrm{C}-\mathrm{NMR}, \delta(\mathrm{ppm}): 116.0$, $116.8,119.9,121.4,121.7,123.1,123.3,123.7,124.3,124.6,124.7,125.2,125.6,125.7,125.8,125.9$, 126.5, 126.6, 127.1, 127.3, 127.6, 127.7, 127.9, 128.4, 128.5, 128.6, 128.7, 129.6, 130.3, 130.6, 133.0, 133.4, 133.6, 133.8, 134.0, 139.0, 140.2, 142.3, 142.5, 142.6, 142.9, 142.9, 143.9, 144.4, 145.1. MS [ESI]: 561 [M+1]. Calcd. for $\mathrm{C}_{34} \mathrm{H}_{24} \mathrm{~S}_{4}: \mathrm{C}, 72.82 ; \mathrm{H}, 4.31 ; \mathrm{S}, 22.87$. Found: C, 72.81; H, 4.32; S, 22.87.

3,4,6,8-tetrakis((E)-2-(thiophen-2-yl)vinyl)azulene-1-carbaldehyde, 23, Dark green crystals, ${ }^{1} \mathrm{H}-$ NMR $\delta(\mathrm{ppm}): 6.94\left(\mathrm{dd},{ }^{3} \mathrm{~J}=5.0 \mathrm{~Hz},{ }^{3} \mathrm{~J}=3.6 \mathrm{~Hz}, 2 \mathrm{H}, 4-\mathrm{H}^{\prime}\right), 6.96\left(\mathrm{dd},{ }^{3} \mathrm{~J}=5.0 \mathrm{~Hz},{ }^{3} \mathrm{~J}=3.6 \mathrm{~Hz}, 1 \mathrm{H}, 4-\right.$ $\left.\mathrm{H}^{\prime \prime}\right), 7.00\left(\mathrm{~d},{ }^{3} \mathrm{~J}=3.6 \mathrm{~Hz}, 1 \mathrm{H}, 3-\mathrm{H}^{\prime}\right), 7.06\left(\mathrm{dd},{ }^{3} \mathrm{~J}=5.0 \mathrm{~Hz},{ }^{3} \mathrm{~J}=3.6 \mathrm{~Hz}, 1 \mathrm{H}, 4-\mathrm{H}^{\prime \prime}\right), 7.07\left(\mathrm{~d}, 1 \mathrm{H},{ }^{3} \mathrm{~J}=\right.$ $\left.16.0 \mathrm{~Hz},=\mathrm{CH}^{\beta}\right), 7.08\left(\mathrm{dd},{ }^{3} \mathrm{~J}=5.1 \mathrm{~Hz},{ }^{4} \mathrm{~J}=3.6 \mathrm{~Hz}, 1 \mathrm{H}, 4-\mathrm{H}^{\mathrm{iv}}\right), 7.15\left(\mathrm{~d},{ }^{3} \mathrm{~J}=5.2 \mathrm{~Hz}, 1 \mathrm{H}, 5-\mathrm{H}^{\prime}\right), 7.17(\mathrm{~d}$, $\left.{ }^{3} \mathrm{~J}=16.2 \mathrm{~Hz}, 1 \mathrm{H},=\mathrm{CH}^{\beta^{\prime \prime}}\right), 7.23\left(\mathrm{~d},{ }^{3} \mathrm{~J}=3.6 \mathrm{~Hz}, 1 \mathrm{H}, 3-\mathrm{H}^{\prime \prime}\right), 7.24\left(\mathrm{~d},{ }^{3} \mathrm{~J}=3.6 \mathrm{~Hz}, 2 \mathrm{H}, 3-\mathrm{H}^{\prime \prime}, 3-\mathrm{H}^{\mathrm{iv}}\right), 7.26$ $\left(\mathrm{d},{ }^{3} \mathrm{~J}=5.2 \mathrm{~Hz}, 1 \mathrm{H}, 5-\mathrm{H}^{\prime \prime}\right), 7.27\left(\mathrm{~d},{ }^{3} \mathrm{~J}=5.0 \mathrm{~Hz}, 2 \mathrm{H}, 5-\mathrm{H}^{\prime}, 5-\mathrm{H}^{\mathrm{iv}}\right), 7.37\left(\mathrm{~d},{ }^{3} \mathrm{~J}=15.9 \mathrm{~Hz}, 2 \mathrm{H},=\mathrm{CH}^{\beta^{3}}\right)$, $7.42\left(\mathrm{~d},{ }^{3} \mathrm{~J}=15.9 \mathrm{~Hz}, 2 \mathrm{H}=\mathrm{CH}^{\beta i v}\right), 7.49\left(\mathrm{~d}, 1 \mathrm{H},{ }^{3} \mathrm{~J}=16.0 \mathrm{~Hz},=\mathrm{CH}^{\alpha}\right), 7.50\left(\mathrm{~d},{ }^{3} \mathrm{~J}=15.9 \mathrm{~Hz}, 1 \mathrm{H}=\mathrm{CH}^{\alpha{ }^{\prime \prime}}\right)$, $7.62(\mathrm{~s}, 2 \mathrm{H}, 5-\mathrm{H}), 7.65(\mathrm{~s}, 1 \mathrm{H}, 7-\mathrm{H}), 7.72\left(\mathrm{~d},{ }^{3} \mathrm{~J}=15.8 \mathrm{~Hz}, 2 \mathrm{H}=\mathrm{CH}^{\beta^{\prime}}\right), 7.76\left(\mathrm{~d},{ }^{3} \mathrm{~J}=15.8 \mathrm{~Hz}, 2 \mathrm{H}\right.$ $\left.=\mathrm{CH}^{\alpha i v}\right), 8.42(\mathrm{~s}, 1 \mathrm{H}, 2-\mathrm{H}), 10.56(\mathrm{~s}, 1 \mathrm{H}, \mathrm{CHO})$. MS [ESI]: 589 [M+1]. Calcd. for $\mathrm{C}_{35} \mathrm{H}_{24} \mathrm{OS}_{4}: \mathrm{C}, 71.39$; H, 4.11; S, 21.78. Found: C, 71.41; H, 4.12; S, 21.75.

\section{Results and discussions Synthesis}

The limited success of sodium N-methylanilide in activating the methyl groups at azulene sevenring for the condensation with thiophene carbaldehyde and in intermediate alcohol dehydration prompted us to change this reagent with potassium tert-butoxide used also by Hansen for arylcarbaldehydes, as presented in the introduction [12]. Another aim of our work was to extend the research form 2-thiophene- to 3-thiophene-carbaldehyde. The latter has the advantage to possess more selective centers for polymerization at free C2 and C5 positions, which allow the formation of dendrimeric compounds after condensation with the azulene derivatives, by oxidative coupling. At the same time, it was expected that under the new reactions conditions, all active methyl groups would react with aldehydes to obtain the polyvinylic azulene compounds. Because guaiazulene has been already extensively investigated (possesses only one active Me group at $\mathrm{C} 4$ ), other active methyl azulenes have now been considered: 6-methylazulene, (which has only one active center in 6-position) 4,8-dimethyl6-tert-butylazulene (possessing two active methyls in positions 4 and 8) and 4,6,8-trimethylazulene (as a tri-center condensing compound).

For the beginning, the reactivity of the Me group in 6-methylazulene was tested. Starting from 2- or 3- thiophenecarbaldehydes, the reaction occurs in the presence of tBuOK, in THF and products 1 or 2 were formed in moderate yields (Scheme 2). Despite its simplicity, the results were lower than in case of guaiazulene. This can be explained by the lower reactivity of C6 anion than that of $\mathrm{C} 4$ anion, which requires a longer reaction time, allowing the formation of by-products. Moreover, 6-methylazulene is susceptible to ring-opening processes through nucleophilic attack, due to the absence of the protecting methyl group at $\mathrm{C} 4(8)$. 


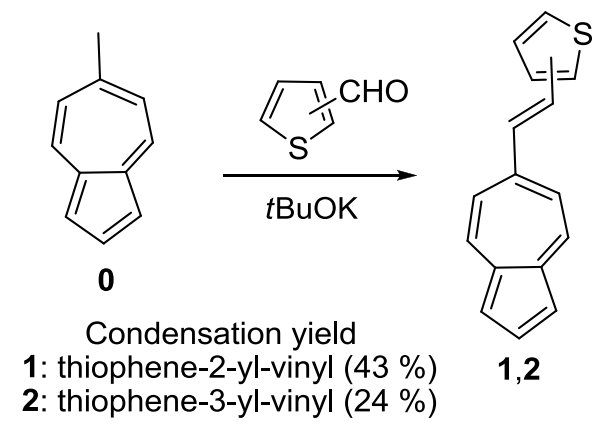

Scheme 2. Reactions of 6-methylazulenes

Condensation of 4,8-dimethyl-6-tert-butylazulene 3, which contains two active methyls in equivalent positions (4 and 8 ) and a bulky inert $t \mathrm{Bu}$ group is more complicated. However, after the first condensation at 4-position, the next position (8) is less reactive due both to electronic and steric factors. Therefore, the rate of the second substitution is slower and the monosubstituted compound can accumulate into the reaction mixture. Moreover, some intermediate alcohol can suffer hydride transfer to the aldehyde in excess. Therefore, the reaction output is different in different buffers: $\mathrm{PhNMeH} / \mathrm{PhNMeNa}$ or $\mathrm{tBuOH} / \mathrm{tBuOK}$, considering the same solvent: mixture of THF and ether. In a previous paper, treating 4,8-dimethyl-6-tert-butylazulene with sodium methylanilide, resulted the mono and bis condensed products in $13 \%$ and $4 \%$ yield, respectively (Scheme 1). These alkenes were accompanied by various ketones in high-yield, as condensed by-products. The results of condensations in the presence of $t$ BuOK shown in Scheme 3 and Table 1 occur when the azulene derivatives reacted almost completely. Higher yields for condensed products and only a moderate amount of ketone $\mathbf{1 8}$ are observed in Scheme 3.

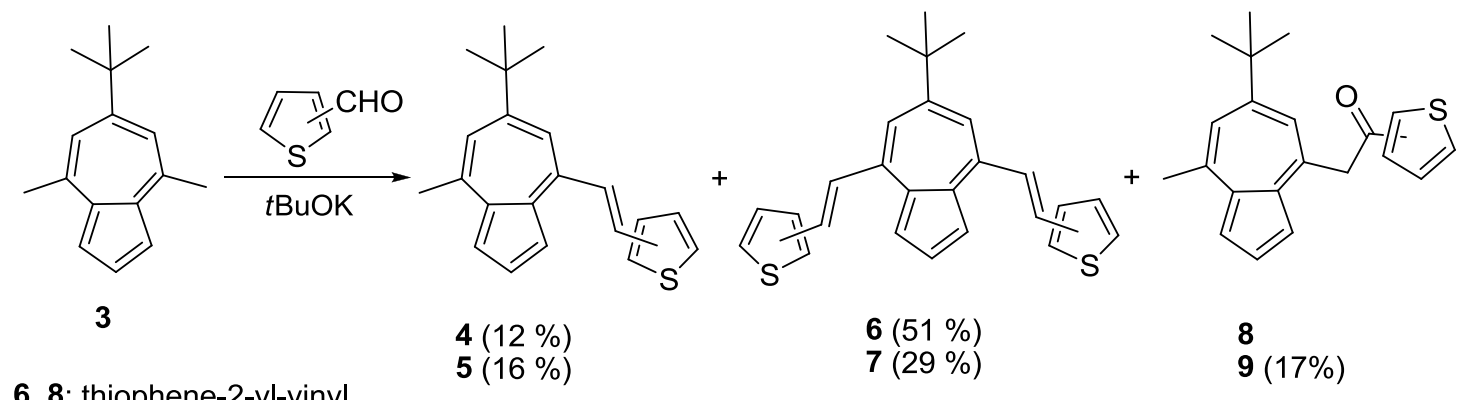

4, 6, 8: thiophene-2-yl-vinyl

5, 7, 9: thiophene-3-yl-vinyl

Scheme 3. Reaction of 4,8-dimethyl-6-tert-butylazulene with thiophenecarbaldehydes

As can be seen from Scheme 4, the amount of oxidized intermediate alcohol can be influenced by the nature of the metal used in the catalytic base. It seems that $\mathrm{Na}^{+}$can form easier the transition state and increases the rate of hydride transfer side reaction. Therefore, more ketones are obtained in the presence of sodium $\mathrm{N}$-methylanilide than in the case of potassium tert-butoxide. The metal involvement in alcohol oxidation by aldehydes is thoroughly studied by Liautard and al. [21].

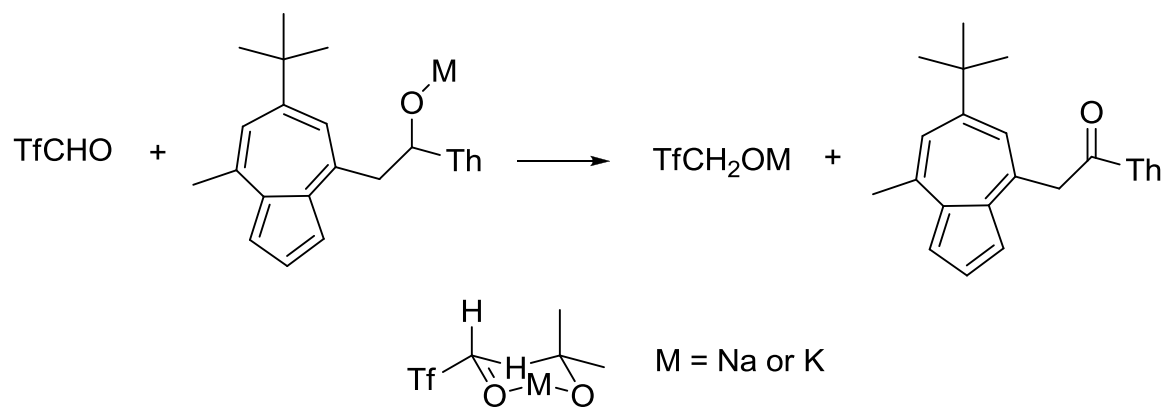

Scheme 4. Hydride transfer from the intermediate alcohols to aldehyde in excess 
The reaction of 4,6,8-trimethylazulene seemed interesting due to the presence of two types of activated methyl groups. Those situated in positions 4 and 8 , close to negatively polarized small cycle, possess a higher acidity than that in 6-position, which is less acidic. Therefore, it is assumed that the kinetic route of reaction favors the condensation in position 4(8) whereas the reaction with methyl substituted at 6-position will lead to more stable alkenes in the thermodynamic control. Our results are labeled a, whereas the products for thiophen-2-yl condensation in the presence of sodium $\mathrm{N}$ methylanilide, which are previously reported [14], are shown on label b in Scheme 5. It is obvious that in the case of potassium t-butoxide, the reaction works better; the yields in bis(thiophenylvinyl) alkenes are increased and tris(thiophenylvinyl) derivatives can be isolated.

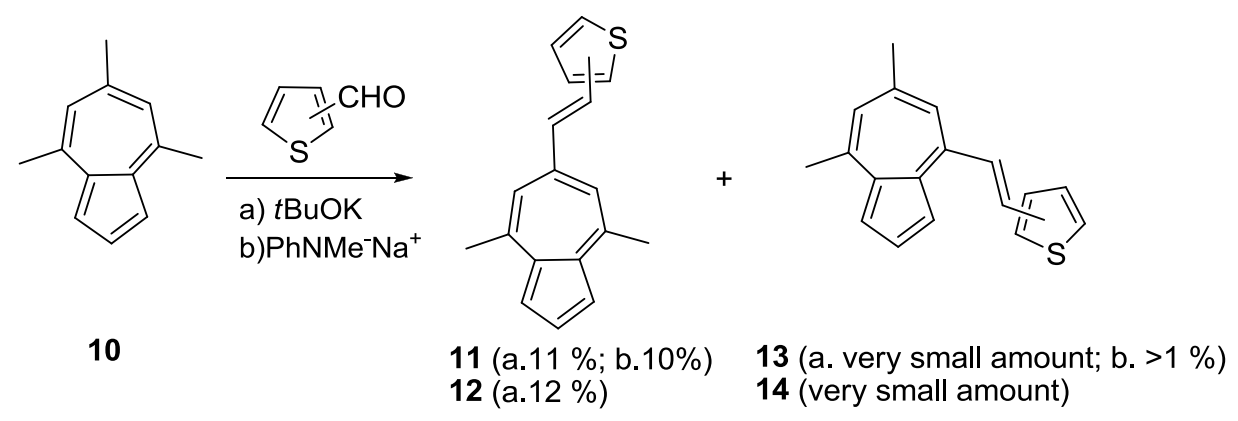

11, 13, 15, 17: thiophene-2-yl-vinyl $12,14,16,18$ : thiophene-3-yl-vinyl

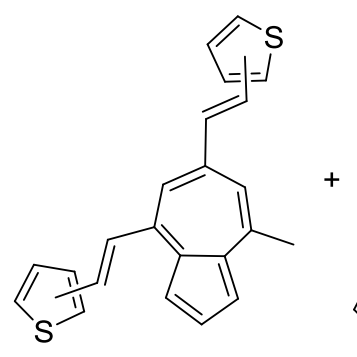

15 (a. $18 \%$; b. $>2 \%$ ) 16 (a. $26 \%$ )

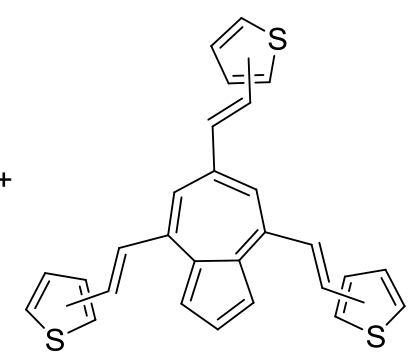

17 (a. $17 \%$; b. not resulted) 18 (a. $15 \%$ )

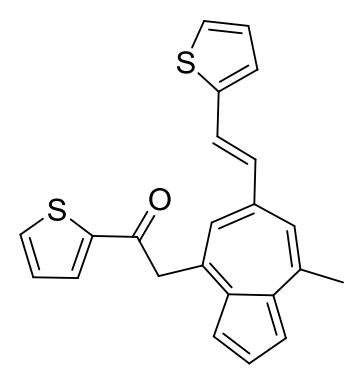

19

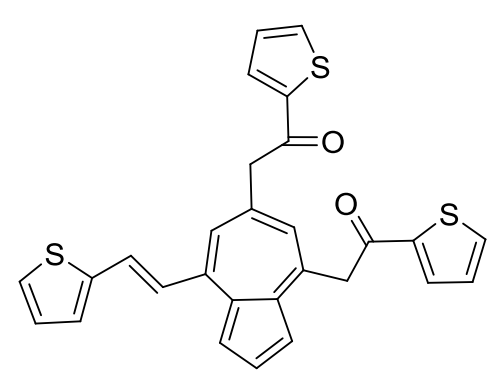

20

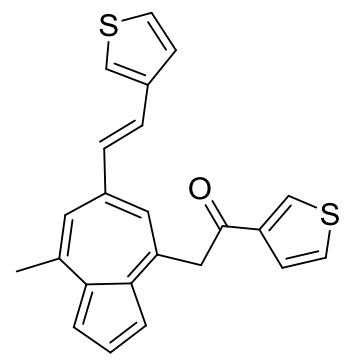

21

Scheme 5. Reaction of 4,6,8-trimethylazulene with thiophenecarbaldehyde

The almost exclusive presence of the condensation product in position 6 in the presence of sodium $N$-methylanilide, as well as when $t \mathrm{BuOK}$ was used, attests the thermodynamic pathway for condensation. The bis condensed products, which are accompanied by those mono-condensed ones, are the major reaction products. Interestingly, only the methyl groups belonging to positions 4 and 6 reacted, while the isomer with double bonds in positions 4 and 8 was not obtained, again proving the thermodynamic control under these conditions. It is noteworthy that among these products, the tricondensed products were generated in a sufficient quantity to be able to be separated and characterized. All products yields, obtained working with both thiophene aldehydes are comparable to each other (Scheme 4) and are better than in the case of PhNMeNa. In the aim to enrich the reaction mixture in the tricondensed product an excess of aldehyde was used. However, similar to what happens 
when sodium $\mathrm{N}$-methylanilide catalyzed the reaction, in addition to a slight increase in the quantity of desired product, a significant amount of by-products resulted, namely ketones. The alcoxide, generated as an intermediate in condensation, reacts with the excess of aldehyde in an Oppenauer oxidation, as presented above. Of these ketones, difficult to separate individually, a few, namely ketones 19-21 in Scheme 5, were still isolated and their structures are compared with these reported in our previous paper [18]. Unfortunately, as by-products in small amounts, several other ketones with high molar masses were highlighted by mass spectrometry. However, their isolation was not our first target or could not be achieved in all experiments for their characterization.

The yields obtained in our condensations with BuOK-THF for the compounds studied are summarized in Table 1-proving the low regioselectivity of the condensations if more active sites are present.

Table 1. Yields of condensation between thiophenecarboxaldehydes and alkylazulenes

\begin{tabular}{|c|c|c|c|c|c|c|}
\hline $\begin{array}{c}\text { Azulene } \\
\text { Substituents }\end{array}$ & Code & & Monoalkene & dialkene & trialkene & Others \\
\hline \multirow[t]{2}{*}{ 6-Me } & $\mathbf{0}$ & $2 \mathrm{TfCHO}$ & 43 & & & \\
\hline & & $3 \mathrm{TfCHO}$ & 24 & & & \\
\hline \multirow[t]{2}{*}{$4,8-\mathrm{Me}_{2}-6-\mathrm{tBu}$} & 3 & $2 \mathrm{TfCHO}$ & 12 & 51 & & KETO \\
\hline & & $3 \mathrm{TfCHO}$ & 16 & 29 & & 17 \\
\hline \multirow[t]{2}{*}{$4,6,8-\mathrm{Me}_{3}$} & 10 & $2 \mathrm{TfCHO}$ & 11 & $18^{*}$ & 17 & KETO \\
\hline & & $3 \mathrm{TfCHO}$ & 12 & $26^{*}$ & 15 & \\
\hline
\end{tabular}

The reaction route for the synthesis of tetra-substituted azulene, 22, described in Scheme 6, consists in the sequence Vilsmeier - Wittig reactions (step 1; yield $63 \%$ ) + (step 2; yield $55 \%$ ). Our attempt to introduce another thiophenyl-vinyl group has been stopped for the time being in aldehyde $\mathbf{2 3}$ stage, but research continues.
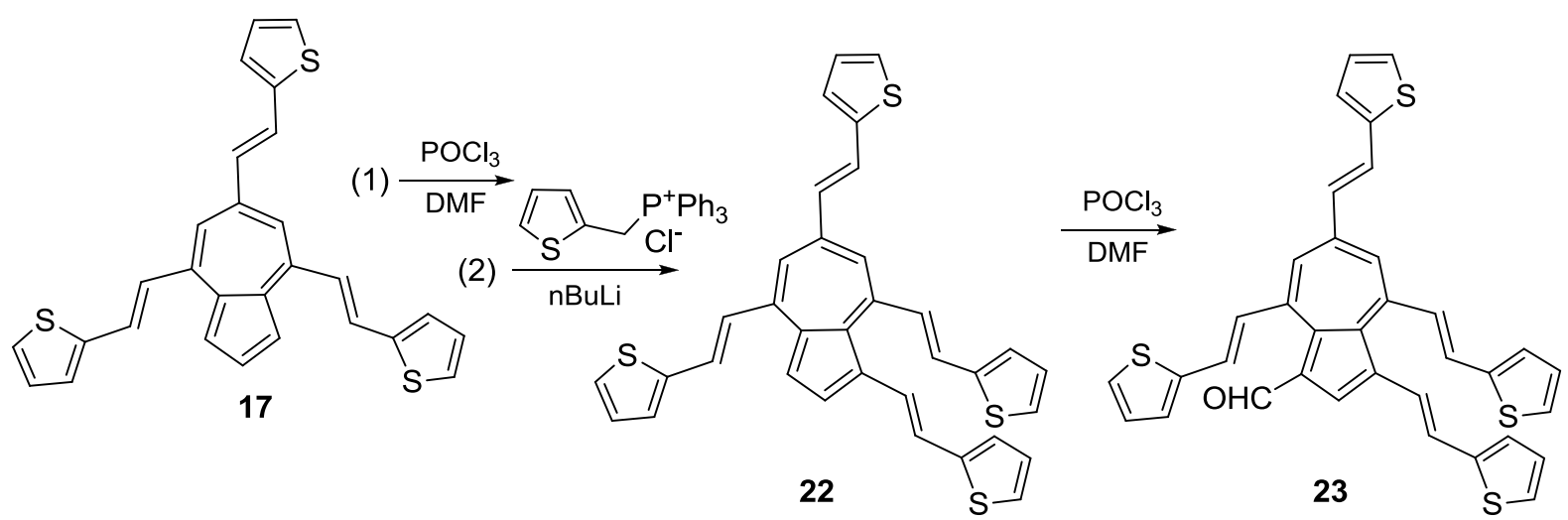

Scheme 6. Preparation of 1,4,6,8-tetra(thiophene-2-ylvinyl)azulenes

\section{Consideration about NMR chemical shifts}

The chemical shifts of the protons are presented in Table 2 and an example of the H-NMR spectrum of 4,6,8-tris(2-thiophenylvinyl)azulene in Figure 1.The most interesting fact is the variation of the vinyl protons. The coupling constants around $16 \mathrm{~Hz}$ show that all compounds have a trans configuration. It can be seen that these are deshielded near the azulene and less deshielded near the thiophene moiety. However, the extent of these influences is very different depending on vinyl group position on the azulene frame. The lowest deshielding is observed for the 6-isomer, which probably sees the lowest anisotropic effect of azulene system. The more shielded vinylic atoms are observed for 1-isomer and the most deshielded for the 4-isomer, which sees the highest anisotropic effect (Scheme 7). 

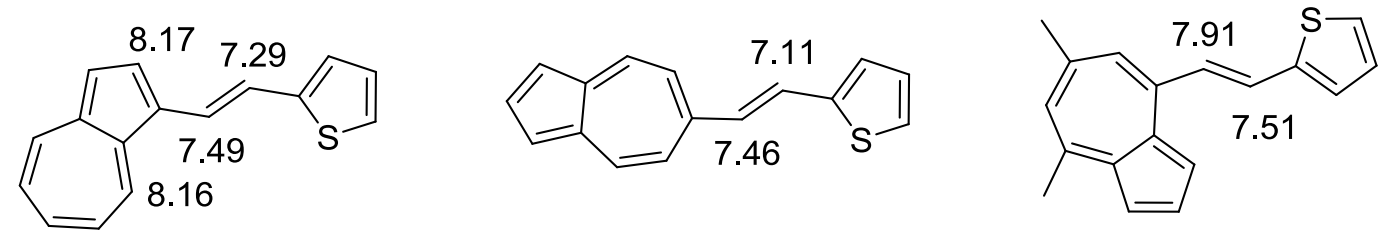

Scheme 7. Proton chemical shifts for vinylic group attached at 1-, 4-, and 6- position

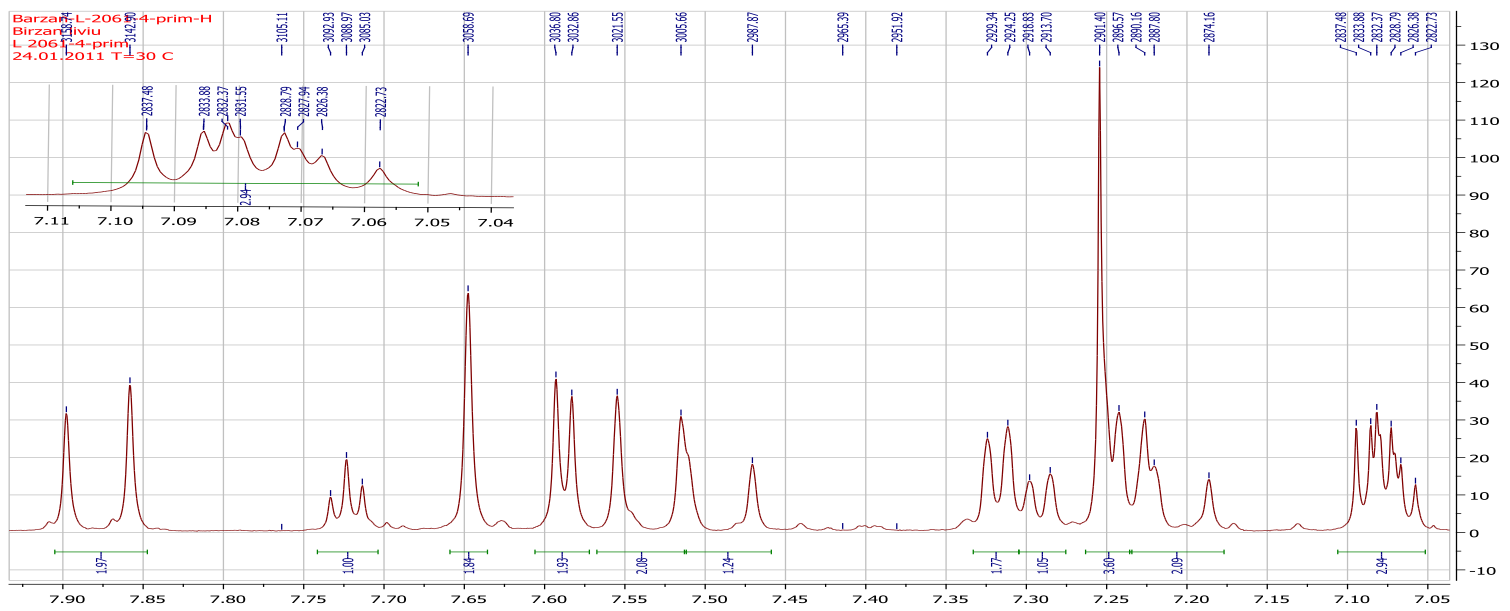

Figure 1. Proton chemical shifts for 4,6,8-tris(2-thiophenylvinyl)azulene

The vinyl group strongly influences the chemical shifts of the azulene protons. The anisotropic effect of the vinyl group induces a deshielding of adjacent azulene protons of about 0.3-0.4 ppm.. However, they are less influenced by the nature of the isomer of the thiophene moiety. However, if the alkyl substituents on the azulenic moiety are identical, the gap between the ethylene protons decreases in the order phenyl $>3$-thienyl $>2$-thienyl, respectively $0.75>0.61>0.44 \mathrm{ppm}$. for the divinylic compounds of 6-tert-butyl-azulene. This leads us to consider that the polarity of the compounds could decrease in the same order, but anisotropy variation is also very important. There are also very small differences between the chemical shifts of the heterocycles situated at the 4-(8-) or 6-position, but the values are different, making the NMR spectra quite complicated. The presence of a keto group linked by the thiophene moiety influences only the chemical shifts of the heterocycle.

The same differences are observed in the spectra of the carbon atoms. The polarization of the molecule is reflected by the gaps between the chemical shifts of vinylic carbons of bis(arylvinyl)-tertbutylazulenes (where aryl is 2-thiophenyl, 3-thiophenyl, phenyl): 3.8, 3.3, 0.2, that meaning that the thiophene moieties are electron richer than azulenic seven-membered cycle. The 2-thiophenyl group is the best electron-donating moiety while benzene ring is very little involved in conjugation. Pushing electrons to the azulene seven-membered cycle, 2-thiophenyl group partially depolarizes azulene reducing the polarity of the molecule.

Table 2. The proton chemical shifts of thiophenylvinylazulene

\begin{tabular}{cccccccccccccccc}
\hline Sub & H1 & H2 & H3 & H4 & H5 & H6 & H7 & H8 & CHTf & CHAz & H2' & H3' & H4' & H5' & Pos \\
\hline & & & & & & & & & & & & & & & \\
A & - & 8.17 & 7.37 & 8.41 & 7.10 & 7.50 & 7.05 & 8.16 & 7.29 & 7.49 & - & 7.07 & 7.00 & 7.15 & 1 \\
B & - & 8.21 & 7.40 & 8.44 & 7.10 & 7.52 & 7.07 & 8.20 & 7.21 & 7.54 & 7.26 & - & 7.45 & 7.34 & 1 \\
C & 7.34 & 7.82 & 7.34 & 8.28 & 7.35 & - & 7.35 & 8.28 & 7.11 & 7.46 & - & 7.18 & 7.05 & 7.28 & 6 \\
D & 7.34 & 7.82 & 7.34 & 8.29 & 7.39 & - & 7.39 & 8.29 & 7.14 & 7.35 & 7.40 & - & 7.41 & 7.37 & 6 \\
E & 7.40 & 7.73 & 7.56 & - & 7.70 & $(1.53)$ & 7.35 & $(2.95)$ & 7.46 & 7.94 & - & 7.22 & 7.08 & 7.31 & 4 \\
F & 7.62 & 7.80 & 7.43 & - & 7.79 & $(1.59)$ & 7.42 & $(3.02)$ & 7.41 & 8.02 & 7.46 & - & 7.56 & 7.45 & 4 \\
G & 7.60 & 7.77 & 7.60 & - & 7.72 & $(1.61)$ & 7.72 & - & 7.48 & 7.92 & - & 7.24 & 7.09 & 7.32 & 4.8 \\
H & 7.62 & 7.79 & 7.62 & - & 7.77 & $(1.61)$ & 7.77 & - & 7.39 & 8.00 & 7.46 & - & 7.54 & 7.41 & 4.8 \\
I & 7.43 & 7.73 & 7.60 & - & 7.45 & $(2.71)$ & 7.10 & $(2.91)$ & 7.51 & 7.91 & - & 7.23 & 7.09 & 7.32 & 4 \\
J & 7.39 & 7.70 & 7.39 & $(2.97)$ & 7.34 & - & 7.34 & $(2.97)$ & 7.11 & 7.46 & - & 7.19 & 7.07 & 7.29 & 6 \\
K & 7.45 & 7.63 & 7.48 & - & 7.56 & - & 7.27 & $(2.95)$ & 7.46 & 7.82 & - & 7.13 & 6.98 & 7.21 & 4
\end{tabular}




\begin{tabular}{|c|c|c|c|c|c|c|c|c|c|c|c|c|c|c|c|}
\hline & & & & & & & & & 7.08 & 7.39 & - & 7.17 & 7.01 & 7.24 & 6 \\
\hline \multirow[t]{2}{*}{$\mathrm{L}$} & 7.59 & 7.73 & 7.59 & - & 7.62 & - & 7.62 & - & 7.15 & 7.48 & - & 7.20 & 7.05 & 7.28 & 6 \\
\hline & & & & & & & & & 7.52 & 7.87 & - & 7.24 & 7.08 & 7.30 & 4,8 \\
\hline M & 7.38 & 7.69 & 7.38 & $(2.96)$ & 7.35 & - & 7.35 & $(2.96)$ & 7.14 & 7.33 & 7.39 & - & 7.41 & 7.35 & 6 \\
\hline \multirow[t]{2}{*}{$\mathrm{N}$} & 7.57 & 7.73 & 7.14 & - & 7.67 & - & 7.36 & $(2.96)$ & 7.18 & 7.35 & 7.41 & - & 7.43 & 7.38 & 6 \\
\hline & & & & & & & & & 7.40 & 7.93 & 7.44 & - & 7.52 & 7.41 & 4 \\
\hline \multirow[t]{2}{*}{$\mathrm{O}$} & 7.58 & 7.72 & 7.58 & - & 7.68 & - & 7.68 & - & 7.24 & 7.37 & 7.42 & - & 7.46 & 7.38 & 6 \\
\hline & & & & & & & & & 7.41 & 7.91 & 7.45 & - & 7.51 & 7.41 & 4,8 \\
\hline \multirow[t]{4}{*}{$\mathrm{P}$} & - & 7.88 & 7.48 & - & 7.42 & - & 7.43 & - & 7.06 & 7.40 & - & 6.97 & 6.92 & 7.07 & 1 \\
\hline & & & & & & & & & 7.08 & 7.69 & - & 7.20 & 7.04 & 7.27 & 6 \\
\hline & & & & & & & & & 7.10 & 7.76 & - & 7.22 & 7.04 & 7.29 & 4 \\
\hline & & & & & & & & & 7.43 & 7.72 & - & 7.24 & 7.08 & 7.31 & 8 \\
\hline
\end{tabular}

$\mathrm{A}=1$-(thiophen-2-ylvinyl)azulene; $\mathrm{B}=1$-(thiophen-3-ylvinyl)azulene; $\mathrm{C}=6$-(thiophen-2-ylvinyl)azulene, 1; $\mathrm{D}=6$-(thiophen-3ylvinyl)azulene, 2; E = 6-(t-butyl)-8-methyl-4-(thiophen-2-ylvinyl)azulene; F = 6-(t-butyl)-8-me-4-(thiophen-3-ylvinyl)azulene, 5; $\mathrm{G}=6$-t-butyl-4,8-bis(thien-2-ylvinyl)azulene; $\mathrm{H}=$ 6-t-butyl-4,8-bis(thiophen-3-ylvinyl)azulene, 7; I = 4-(thiophen-2-ylvinyl)-6,8dimethylazulene; $\mathrm{J}=6$-(thiophen-2-ylvinyl)-4,8-dimethyl-azulene; $\mathrm{K}=4$,6-bis(thiophen-2-ylvinyl)-8-methyl-azulene; $\mathrm{L}=4,6,8$ tris(thien-2-ylvinyl)azulene; $\mathrm{M}=6$-(thiophen-3-ylvinyl)-4,8-methyl-azulene, 12; $\mathrm{N}=4$,6-bis(thiophen-3-ylvinyl)-8-methyl-azulene, 16; $\mathrm{O}=4,6,8$-tris(thiophen-3-ylvinyl)azulene, 18; $\mathrm{P}=1,4,6,8$-tetra(thien-2-ylvinyl)azulene 22. Some data are taken from [18-20]

\section{Electronic spectra}

These thiophenylvinylazulenes possess several UV-Vis bands. For their color determination are important two bands: the major visible band, representing $\mathrm{S}_{2}-\mathrm{S}_{0}$ transition between LUMO+1 and HOMO orbitals, which is allowed by the orbital geometries and the second band, representing $\mathrm{S}_{1}-\mathrm{S}_{0}$ transition, between LUMO and HOMO, which is forbidden by orbitals geometry. Therefore, the last band is very weak, and is divided in a plethora of mini-bands, which are grouped in function of the solvent concentration; therefore, they are not presented but they influence the compounds color. Table 3 shows that the thiophenylvinyl groups induce bathochromic effects on the azulene moiety. This effect is higher for the first group attached in the position 4, 6 or 8 and decreases for the following attached groups. The 6-position induces the greatest effect, because no steric effect is present to prevent the coplanarity of the systems, as is expected for 4(8)-positions. The 2-thiophenyl moiety is more powerful than 3-thiophenyl; for example, in the case of 6-isomer, the main visible bands are at $407 \mathrm{~nm}$ and 392 $\mathrm{nm}$, respectively. The presence of methyl groups in other position does not influence significantly the chromophore, as in the case of $\mathbf{J}$ and $\mathrm{M}$. The thiophenylvinyl group in 4-position has a lower impact on the visible band; in $\mathrm{H}$ the absorption maximum is only $377 \mathrm{~nm}$. However, if two such 3-thienylvinyl groups are present in 4- and 6- only that of 6-position influences, in fact, the chromophore. Even in the case of three 2-thiophenylvinyl groups in 4,- 6-, and 8- positions only a minor bathochromic shift of 8 $\mathrm{nm}$ is observed compared with 6-thien-2-ylvinyl chromophore and the situation is similar for 6-thiophen3 -ylvinyl isomer. The presence of four thiophen-2-ylvinyl groups in 1,4,6,8-positions presents the most bathochromic maxima of absorption of $418 \mathrm{~nm}$.

Table 3. UV-Vis spectra of some thienylvinylazulenes (bands III and IV)

\begin{tabular}{cccccccccccc}
\hline A & C & D & J & E & F & H & L & M & N & O & P \\
\hline $314 / 318$ & 328 & 315 & 328 & 336 & 332 & 335 & 366 & 322 & 332 & 347 & \\
$393 / 413$ & 407 & 392 & 407 & 360 & 388 & 377 & 410 & 390 & 388 & 392 & 418 \\
\hline
\end{tabular}

The labels are the same as above (Table 2) Some data are taken from [18-20]

\section{Conclusions}

Some conclusions can be drawn based on the condensation reactions described above. Depending on the structure of the azulene used as starting material, the molar ratio between azulene derivatives and thiophenecarbaldehydes, catalyst, solvent, the reaction mixtures may contain mono-, bis- and triscondensation products. The attempt to obtain only the fully condensed products in the presence of high aldehyde excess failed. The reaction mixtures remain constant at high aldehyde excesses proving the reactions reversibility. The exclusive or prevailing condensation at position 6 as compared with that at position 4(8) validates the reaction thermodynamic control. Moreover, all the condensation compounds 
are trans. Because the removal of water from intermediate alcohols takes time, an amount of the alcohols can transfer hydride to the excess of aldehyde forming ketones. The nature of the cation of the base catalyst is possible to influence the amount of the undesired ketones formed.

Further attempts were made to increase the number of thiophenyl-vinyl groups attached to the azulene. The methyl groups placed on the small-ring are not acidic enough to be ionized by strong bases; therefore, the reaction with aldehydes was not possible until the methyl groups was activated by inserting ${ }^{+} \mathrm{PPh}_{3}$ group. Formylation of thiophenevinylazulenes works in Vilsmeier conditions in good yields.

\section{References}

1. RAZUS, A. C. J. Chem. Soc., Perkin Trans., 1, 2000, p. 981.

2. RAZUS, A. C., BIRZAN, L., NAE, S., RAZUS, S. A., CIMPEANU, V., STANCIU, C. Synth. Commun., 32, 2002, p. 825.

3. RAZUS, A. C., BIRZAN, L., NAE, S., SURUGIU N. M., CIMPEANU, V. J. Heterocycl. Chem., 40, 2003, p. 995.

4. RAZUS, A. C., BIRZAN, L., CRISTEA, M., TECUCEANU, V., DRAGHICI, C., HANGANU, A., MAGANU, M., PINTILIE, L., UNGUREANU, E. M., Rev. Chim., 70(5), 2019, p. 1518.

5. RAZUS, A. C., BIRZAN, L., CRISTEA, M., TECUCEANU, V., DRAGHICI C., Rev. Chim., 66, 2015, p. 1074.

6. RAZUS, A. C., NITU, C., CARVACI S,, L. BIRZAN, RAZUS, S. A., POP M., TARKO L., J. Chem. Soc., Perkin Trans., 1, 2001, p. 1227.

7. RAZUS, A. C., NITU, C., TECUCEANU V. CIMPEANU, V. Eur. J. Org. Chem., 2003, 4601.

8. RAZUS, A. C., BIRZAN, L. Arkivoc, iv, 2018, p. 1.

9. ZELLER K. P., IN METHODEN DER ORGANISCHEN CHEMIE; G. THIEME VERLAG: STUTTGART, NEW YORK, 1985; Vol. V/2c, p. 127-416.

10. SHOJI, T., ITO, S. Adv. Heterocycl. Chem. 126, 2018, p. 1.

11. CRISTIAN, L., SASAKI, I., LACROIX, P. L.; DONNADIEU, B., ASSELBERGHS, I., CLAYS K., RAZUS A. C. Chem. Mater., 16, 2004, p. 3543.

12. UNGUREANU, E.-M., RAZUS, A. C., BIRZAN, L., BUICA, G., CRETU M., ENACHE, C. Electrochim. Acta, 52, 2006, p. 794.

13. HORINO, H., ASAO, T., INOUE, N. Bull. Soc. Chim. Jpn., 64, 1991, p. 183.

14 SCHOLZ, M., VIEN, L., FISCHER, G., TSCHAPKE, B., MUHLSTADT M. Chem. Ber. 100, 1967, p. 315.

15. SONG, J., HANSEN H.-J. Helv. Chim. Acta, 82, 1999, p. 309.

16. GHAZVINI ZADEH, E. H., TANG, S., WOODWARD, A. W., LIU, T., BONDAR, M. V., BELFIELD, K. D. J. Mater. Chem. C, 3, 2015, p. 8495.

17. BRIQUET, A. A. S., HANSEN H.-J. Helv. Chim. Acta, 77, 1994, p. 1921.

18. RAZUS, A. C., BIRZAN, L., TECUCEANU, V., CRISTEA, M., ENACHE, C. Arkivoc, xi, 2008, p. 210.

19. RAZUS, A. C., NITU, C., TECUCEANU V. CIMPEANU, V. Eur. J. Org. Chem., 2003, p. 4601. 20. RAZUS, A. C., BIRZAN, L. TECUCEANU, V. CRISTEA, M., NICOLESCU A., ENACHE, C. Rev. Roum. Chim. 52, 2007, p. 189.

21. AMIR, E., AMIR, R. J., CAMPOS L. M., HAWKER, C. J., J. Am. Chem. Soc., 133, 2011, p. 10046. 22. LIAUTARD, V., BIREPINTE, M., BETTOLI, C., PUCHEAULT M. Catalysts, 8, 2018, p. 529

Manuscript received: 29.05 .2020 